\title{
Evolution of Cold-Air-Pooling Processes in Complex Terrain
}

\author{
Paul Burns · Charles Chemel
}

Received: 18 July 2013 / Revised: R1 27 September 2013, R2 24 October 2013

\begin{abstract}
Elucidating cold-air-pooling processes forms part of the longstanding problem of parametrizing the effects of complex terrain in larger-scale numerical models. The Weather Research and Forecasting model has been set-up and run at high resolution over an idealized alpine-valley domain with a width of order $10 \mathrm{~km}$, to investigate the four-dimensional variation of key cold-air-pooling forcing mechanisms, under decoupled stable conditions. Results of the simulation indicated that the total average valley-atmosphere cooling is driven by a complex balance/interplay between radiation and dynamics effects. Three fairly distinct regimes in the evolution of cold-air-pooling processes have been identified. Starting about $1 \mathrm{hr}$ before sunset, there is an initial 30-min period when the downslope flows are initiated and the total average valley-atmosphere cooling is dominated by radiative heat loss. A period of instability follows, when there is a competition between radiation and dynamics effects, lasting some $90 \mathrm{~min}$. Finally, there is a gradual reduction of the contribution of radiative cooling from 75 to $37 \%$. The maximum cold-air-pool intensity corresponds to the time of minimum radiative cooling, within the period of instability. Although, once the flow is established, the valley atmosphere cools at broadly similar rates by radiation and dynamics effects, overall, radiation effects dominate the total average valley-atmosphere cooling. Some of the intricacies of the valley mixing have been revealed. There are places where the dynamics dominate the cooling and radiation effects are minor. Characteristics of internal gravity waves propagating away from the slopes are discussed.
\end{abstract}

Keywords Cold-air pools · Downslope flows · Numerical simulation · Radiative heat loss

P. Burns

Centre for Atmospheric \& Instrumentation Research (CAIR), University of Hertfordshire, College Lane, Hatfield, AL10 9AB, UK

C. Chemel (ه)

National Centre for Atmospheric Science (NCAS), Centre for Atmospheric \& Instrumentation Research (CAIR), University of Hertfordshire, College Lane, Hatfield, AL10 9AB, UK

e-mail: c.chemel@herts.ac.uk 


\section{Introduction}

There is a need to understand and accurately model atmospheric processes in hilly and mountainous terrain (i.e., complex terrain). More specifically, accurate simulations are important, for example, for effective weather and storm predictions, road transportation and aviation safety, as well as for the agricultural industry (e.g. Price et al. 2011). Accurate simulations are required at the local scale for the prediction of air quality (Anquetin et al. 1999; Brulfert et al. 2005; Chazette et al. 2005; Szintai et al. 2010), avalanches (Lundquist 2010), wild and prescribed fires, for impact assessments for proposed new settlements and structures (Zardi and Whiteman 2013), and for climate change estimates (Daly et al. 2010). Regions of complex terrain are also thought capable of affecting the evolution of atmospheric systems on a wider scale (Noppel and Fiedler 2002; Price et al. 2011).

For the foreseeable future, the representation of the effects of complex terrain, in both high-resolution forecast models and low-resolution climate and earth-system models, is likely to require varying levels of parametrization, which requires a sound understanding of the underlying physical processes. One key process in complex terrain is cold-air pooling, ultimately driven by a net loss of longwave radiation from the ground surface to space, typically during nocturnal hours and the winter season. Cold air pools (CAPs) can create large temperature variations over short distances in even small-scale terrain. For example, Gustavsson et al. (1998) reported near-surface air temperature variations of approximately $7 \mathrm{~K}$ over length scales of order $1 \mathrm{~km}$, in terrain with elevation variations less than $100 \mathrm{~m}$, where in some places temperatures decreased by $8.5 \mathrm{~K}$ in $1 \mathrm{hr}$ from sunset. Such temperature variations are currently not well represented in forecast models (Price et al. 2011).

The present work considers cold-air-pooling processes in a valley atmosphere that is not subject to any synoptic forcing, which approximates the case of weak synoptic flows, or where the valley atmosphere is shielded from larger-scale flows by the terrain and possibly a stable layer. In these conditions the local weather and climate are driven by downslope flows and in situ cooling (Whiteman 2000), but uncertainty remains over their respective contributions and their variations in space and time (Price et al. 2011).

Previous observational and modelling studies have described characteristics of CAPs in relation to their environment (see Zardi and Whiteman 2013, for a review, and references therein). However, these studies have generally not focused on quantifying the respective contributions of CAP forcing mechanisms. Several measurement campaigns have aimed at elucidating cold-air-pooling processes for broadly similar mid-latitude climates and conditions (e.g. Price et al. 2011; Sheridan et al. 2013). Price et al. (2011) argued that the dominant process is in situ cooling for small-scale valleys (i.e., valleys about $100-\mathrm{m}$ deep and 1 - to $3-\mathrm{km}$ wide). The argument is that the valley air is decoupled from the atmosphere above, due to the sheltering effect of the valley geometry, reducing turbulence within the valley and preventing heat transfer from above, allowing the valley atmosphere to cool by radiative heat loss to a greater degree than on more exposed ground. The coupling between atmospheric stability and turbulence is made clear, however, a detailed investigation into the characteristics of downslope flows and the valley radiation field was not made. Thompson (1986) used wind and temperature observations collected from Utah, USA and Ontario, Canada, to argue that downslope flows were not the cause of CAPs found in valleys of a very similar scale to those investigated by Price et al. (2011). Thompson (1986) indicated that accurate observations made with bi-directional wind vanes positioned $0.3 \mathrm{~m}$ above ground level, targeted at detecting any downslope flow, did not detect any flows. However, no detailed information about the equipment was provided, and given the terrain over which the atmosphere was measured, and the low heights of the instruments above ground level, it is possible that the 
wind speeds were close to the threshold values of the wind vanes. The Utah measurements found that valley flows began after the development of the valley temperature inversion suggesting that downslope flows were not the cause of it. However, weak downslope flows could have contributed to the development of the valley temperature inversion in the first instance. Ambient wind-speed data was not provided, and the stability of the atmosphere was not discussed. The site characteristics, such as land use, surface roughness, soil type, and moisture content were not considered, although Gustavsson et al. (1998) provided evidence that suggests these latter variables have only a modulating effect on the formation of CAPs.

In contrast to the conclusions of Price et al. (2011) and Thompson (1986), Gustavsson et al. (1998), who made measurements in similar terrain to the former two studies, in southwestern Sweden, pointed out that downslope flows can be important for the development of CAPs. The lateral extent of the observed CAPs was found to increase during the night. The dependence of this lateral expansion on valley width and drainage area was clearly shown. However, without further investigation, it is difficult to assert that this lateral expansion was due to downslope flows rather than due to radiation effects. Gustavsson et al. (1998) found a strong correlation between valley drainage area and the strength of the CAP, measured by comparing near-surface air temperatures, and also demonstrated the complicating effects of forested regions on cold-air pooling, the tree canopy apparently enhancing the cooling process due to a sheltering effect.

There have been a number of numerical modelling works focused on improving our understanding of downslope flows and cold-air-pooling processes (e.g. Anquetin et al. 1998; Skyllingstad 2003; Smith and Skyllingstad 2005; Vosper and Brown 2008; Catalano and Cenedese 2010; Smith et al. 2010; Vosper et al. 2013). Hoch et al. (2011) used the MYSTIC (Monte Carlo code for the physically correct tracing of photons in cloudy atmospheres) code (Mayer and Kylling 2005; Mayer 2009), which accounts for inhomogeneous surface albedo and topography, to investigate longwave radiation heating and cooling rates in different topographies. The accuracy of the results is dependent on the assumed atmospheric temperature profiles and simplified ground-air temperature differences. Contributions to heating rates from dynamical processes was not explicitly investigated. The nocturnal radiative contribution to cooling rates was investigated by comparing MYSTIC-computed averagebasin-atmosphere cooling rates in the Arizona meteor crater, USA, to the observed averagebasin-atmosphere total temperature tendency. The observed total rates were estimated by constructing hourly vertical temperature profiles from a meteorological station on the crater floor, time-interpolated 3-hourly tethersonde and radiosonde launches, and a mid-latitude standard atmosphere above $20 \mathrm{~km}$, beyond the range of the radiosonde system. Horizontal uniformity was assumed, based on previous measurements in the crater. The vertical profiles were also used as initial conditions for the MYSTIC simulations, which assumed a rotationally symmetric crater geometry to reduce computational time. Average basin heating and cooling rates were calculated by weighting the vertical profile points according to the proportion of the basin volume they represented. Hoch et al. (2011) found that the radiative contribution, defined above, averaged over one night, was $28 \%$. The percentage contribution reached a maximum value of $75 \%$ shortly before sunrise when wind speeds were low. A minimum percentage contribution of $9 \%$ occurred during an air intrusion into the basin atmosphere in the middle of the night. The accumulated radiative cooling contribution was found to decrease from approximately 30 to $22 \%$ during the course of the night. These latter values were found to lie within a factor of three of comparable estimates of a few earlier studies (see Hoch et al. 2011, and references therein). The crater is approximately $150 \mathrm{~m}$ deep and $1.2 \mathrm{~km}$ across, and so has a very similar scale to the terrain investigated by Gustavsson et al. (1998) and Price et al. (2011). There is a clear difference in geometries, however, Hoch 
et al. (2011) did not find any large difference in cooling rates between valleys and basins of similar scales and under similar atmospheric and boundary conditions. The Arizona meteor crater lies at about $30{ }^{\circ} \mathrm{N}$ and has a semi-arid climate. The lack of moisture close to the ground/air interface is likely to enhance the ground-air temperature excesses and deficits relative to more northerly regions, where a greater portion of the available energy is stored as latent heat (Hoch et al. 2011).

Despite considerable effort and progress, it is apparent that uncertainty remains about the physical processes controlling CAPs. Detailed investigations of these processes are needed. In the present work, a numerical model is used to examine the variation of key cold-airpooling forcing mechanisms in an idealized alpine-valley domain with a width of order $10 \mathrm{~km}$ under decoupled stable conditions. The set-up of the model and the design of the numerical simulation are presented in Sect. 2. Numerical results are analyzed in Sect. 3 and a summary is given in Sect. 4 .

\section{Design of the numerical simulation}

The numerical simulation presented herein was performed with the Weather Research and Forecasting (WRF) model (Skamarock et al. 2008), version 3.4.1. The WRF model is specifically designed for research and operational forecasting on a range of scales.

\subsection{WRF numerical formulation}

The WRF model is a fully compressible and non-hydrostatic model that uses a terrainfollowing hydrostatic-pressure vertical coordinate with a constant pressure surface at the top of the domain and a staggered grid of type Arakawa-C. A number of dynamics options are available (see Skamarock et al. 2008, for details). For the present work, time integration is performed using a third-order Runge-Kutta scheme using a mode-splitting time integration technique to deal with the acoustic modes. Momentum and scalar variables are advected using a fifth-order Weighted Essentially Non-Oscillatory (WENO) scheme with a positive definite filter (Shu 2003) with no artificial diffusion. Here, the valley atmosphere is not subjected to any synoptic forcing, and so the relevant Rossby number is that based on the downslope flow, that is $R o=U /(f L)$, where $U$ and $L$ are the typical velocity and length scales of the downslope flow and $f$ is the Coriolis parameter. Given the scales of the problem $\left(U \approx 2 \mathrm{~m} \mathrm{~s}^{-1}, L \approx 3 \mathrm{~km}\right.$ and $f \approx 10^{-4} \mathrm{~s}^{-1}$, see Sect. 2.2 and 3.2.1), the Rossby number is $\gg 1(R o \approx 7)$, and so Coriolis effects were neglected by setting $f=0$.

The model was run in a large-eddy simulation (LES) mode (i.e., with no boundary-layer parametrization scheme) with a vertical grid resolution $\Delta z$ selected to capture the downslope flows (see also Sect. 2.3). The vertical length scale of the downslope flows is given by the height of the wind maximum, denoted by $n_{j}$ hereafter. For the relatively steep slopes of the terrain considered here (see Sect. 2.2), $n_{j}$ is expected to be of order 1-10 m. This range was drawn from appropriate observational studies (e.g. Doran and Horst 1983; Helmis and Papadopoulos 1996). To minimize errors due to large grid-cell aspect ratios, a high horizontal resolution is therefore required (see Sect. 2.3). A turbulent kinetic energy 1.5-order closure scheme (Deardorff 1980) was used to model the subgrid scales. The constant ' $C_{k}$ ' in the subgrid-scale parametrization scheme was set to 0.10 (see Moeng et al. 2007). Because of the anisotropy of the grid, the width of the filter for the subgrid scales was modified following Scotti et al. (1993) (see also Catalano and Cenedese 2010). 
The WRF model includes a number of physics modules, which have a number of formulations that can be selected. The physics schemes used for this work are listed below.

- The Dudhia (1989) scheme was chosen to represent shortwave radiation processes. The scheme performs downward integration of solar flux, accounting for clear-air scattering, water vapour absorption (Lacis and Hansen 1974), and cloud albedo and absorption, using look-up tables for clouds from Stephens (1978). Slope effects on the surface solar flux, and slope shadowing effects, were deactivated. As well as simplifying the problem, this allows for later investigation into the importance of these effects.

- The Rapid Radiation Transfer Model (RRTM) was chosen to represent longwave radiation processes. This spectral-band scheme uses the correlated-k method (Iacono et al. 2008), and pre-set tables to accurately represent the effects of water vapour, carbon dioxide, ozone, methane, nitrous oxide, oxygen, nitrogen and halocarbons. The two radiation schemes were called every minute, a compromise between the need to keep computational time within acceptable limits, and the need to update radiation variables on a time scale similar to the typical time scale over which these variables change significantly. Both schemes were set to account for the impact of clouds on optical depths.

- The National Severe Storms Laboratory (NSSL) two-moment microphysics scheme was selected. The scheme predicts the mass mixing ratio and number concentration for six hydrometeor species: cloud droplets, rain drops, ice crystals, snow, graupel, and hail (see Mansell et al. 2010). The scheme is intended for cloud-resolving simulations where the horizontal resolution $\Delta x$ is less than $2 \mathrm{~km}$.

- The revised MM5 Monin-Obukhov surface-layer scheme by Jiménez et al. (2012) was chosen. The scheme uses the similarity functions of Cheng and Brutsaert (2005) and Fairall et al. (1996), which are suitable under strongly stable and unstable conditions, respectively. Both similarity functions range from neutral conditions, enabling the full range of atmospheric stabilities to be accounted for. Momentum fluxes are calculated by the surface-layer scheme, which also calculates exchange coefficients for momentum, heat and moisture $\left(C_{d}, C_{h}\right.$ and $C_{q}$, respectively) that are passed to the specified landsurface model (LSM), which then calculates the surface fluxes of heat and moisture. The thermal roughness length $z_{0 h}$, over land surfaces, was set to depend on vegetation height rather than being set constant. Since $z_{0 h}$ helps to determine $C_{h}$ and $C_{q}$, this leads to a more accurate representation of surface-atmosphere interactions (Chen and Zhang 2009). The Obukhov length scale, $L_{O}$, is used to scale the fluxes. Although friction acts at inclined surfaces, turbulence production is dominated by the downslope flow wind maximum at $n_{j}$, which is the relevant length scale with which to scale the fluxes (Grisogono et al. 2007). Turbulence above the wind maximum is decoupled from the surface (Zardi and Whiteman 2013). Whenever $L_{O}>n_{j}$, the length scale of the turbulent eddies that determine the fluxes is not the most relevant length scale. Grisogono et al. (2007) demonstrated that this is more likely to occur as the slope angle and/or stratification are increased. However, for the present work, $L_{O} \leq n_{j}$ (not shown).

- The community Noah LSM (Chen and Dudhia 2001) was chosen with four soil layers. The United States Geological Survey (USGS) land-use table was chosen, which provides 24 different land-use categories, and 16 soil categories were included. Both the land-use and soil category were set constant across the model domain (see Sect. 2.4). 


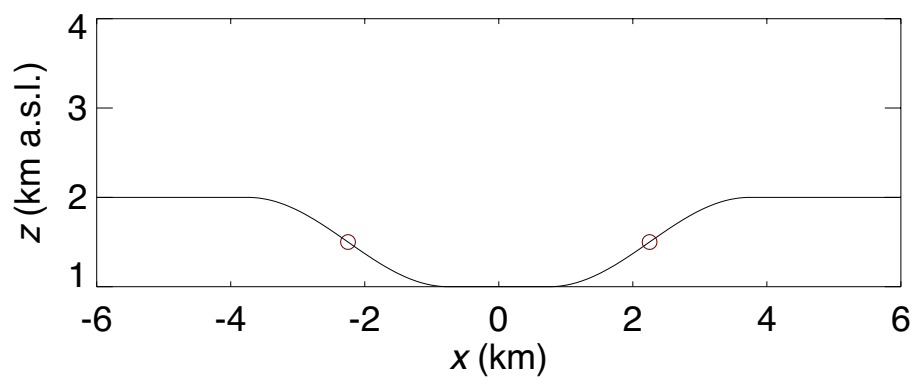

Fig. 1 Terrain height. The red circles mark the slope inflection points. The terrain is uniform along $y$ (into the page), though $y$ was given a length of $1.2 \mathrm{~km}$.

\subsection{Idealized terrain}

An idealized U-shaped valley, with its axis orientated north-south, was implemented with a maximum slope angle of $27.6^{\circ}$, flanked on either side by a horizontal plateau extending $2.25 \mathrm{~km}$ from the top of the valley slopes. The terrain height above sea level (a.s.l.) is given by

$$
h(x, y)=H h_{x}(x) h_{y}(y)+z_{\text {ref }},
$$

where $x$ and $y$ are the west-east and south-north components of the model curvilinear coordinate system, respectively, $H$ is the maximum depth of the valley, $z_{\text {ref }}$ is the height of the bottom of the valley, and $h_{x}(x)$ and $h_{y}(y)$ are defined as

$$
h_{x}(x)=\left\{\begin{array}{ll}
0.5\left\{1-\cos \left[\pi\left(\left|x-V_{x}\right|\right) / S_{x}\right]\right\}, & V_{x} \leq|x| \leq S_{x}+V_{x} \\
0, & |x|<V_{x} \\
1, & |x|>S_{x}+V_{x}
\end{array},\right.
$$

and

$$
h_{y}(y)=0.5\left[1+\tanh \left(y / S_{y}\right)\right],
$$

where $V_{x}$ is the half width of the valley floor, $S_{x}$ is the $x$-dimension slope length, and $S_{y}$ is the $y$-dimension slope length. To simplify the problem, $h_{y}(y)$ was held constant at unity, making the topography uniform in $y$, though $y$ was given a length of $1.2 \mathrm{~km}$. We set $z_{\text {ref }}=1000 \mathrm{~m}$, $H=1000 \mathrm{~m}, V_{x}=750 \mathrm{~m}$ and $S_{x}=3000 \mathrm{~m}$. These values approximate the environment of the lower Chamonix Valley, located in the French Alps $\left(45.92{ }^{\circ} \mathrm{N}, 6.87^{\circ} \mathrm{E}\right)$ and all model points were assigned these coordinates. Figure 1 illustrates the geometry of the terrain.

\subsection{Model grid}

The model was discretized using 101 staggered grid points along the $z$-direction. The vertical coordinate (defined by $\eta$ levels) was stretched using a hyperbolic tangent function, from Vinokur (1980), defined by

$$
\eta(k)=-\tanh \left[A\left(\frac{k-1}{k_{\max }-1}-1\right)\right] / \tanh (A),
$$

where $k$ is the vertical staggered grid point number (ranging from 1 to $k_{\max }=101$ ), and $A$ is a coefficient used to adjust the stretching, given a value of 3.134, with larger $A$ values 


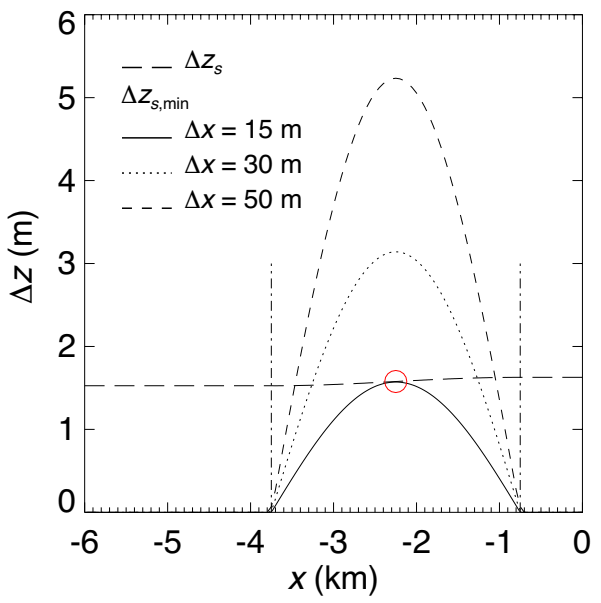

Fig. 2 Comparison of the vertical grid resolution adjacent to the ground surface, denoted by $\Delta z_{s}$, against the minimum acceptable $\Delta z_{s}$, denoted by $\Delta z_{s, \text { min }}$, given by Eq. 5 with $b=5$, for three values of horizontal resolution $\Delta x$. The vertical dot-dashed lines mark the start and end of the western slope, and the red circle marks the position of the slope inflection point.

providing greater vertical grid resolutions. This function provides decreasing resolution with increasing $z$, with a model top located at $12 \mathrm{~km}$ a.s.l., and $\Delta z$ values adjacent to the ground surface, denoted by $\Delta z_{s}$, of approximately 1.52 and $1.62 \mathrm{~m}$ over the plateaus and valley floor, respectively, with $\Delta z_{s}$ ranging between these two limits over the slopes of the valley. To obtain numerically stable results, this range of $\Delta z_{s}$ values demanded a model time $\Delta t=$ $0.05 \mathrm{~s}$. The acoustic timestep was set equal to $\Delta t / 10$. Given the relatively steep slopes of the terrain considered here (see Sect. 2.2), the parameter ' $\beta$ ', used in the model to damp vertically propagating sound waves, was set to 0.9 (see Dudhia 1995).

Mahrer (1984) demonstrated that, when using a terrain-following coordinate system, errors in the approximation of horizontal gradients are more likely to occur at large grid-cell aspect ratios, when the lengths of the grid cells are larger than their heights. This makes it more common for the change in $z$, caused by moving between neighbouring $x$ points (holding $\eta$ constant), denoted by $\Delta z_{\Delta x}$, to be larger than the vertical resolution $\Delta z$. To avoid errors in the approximations of horizontal gradients, $\Delta z$ should be set so that $\Delta z \geq \Delta z_{\Delta x}$. Noting that $\Delta z_{\Delta x}=\Delta x \tan |\alpha|$ at the ground surface, where $\alpha$ is the slope angle, and introducing a parameter $b$ allowing for a range of acceptable grid cell distortions, this gives

$$
|\alpha| \leq \arctan \left(b \frac{\Delta z_{s}}{\Delta x}\right)
$$

Equation 5 can be used to estimate the minimum $\Delta z_{s}$ for given values of $\alpha$ and $\Delta x$. The parameter $b$ is commonly set in the range 1-5, with 5 providing the minimum acceptable $\Delta z_{s}$, denoted by $\Delta z_{s, \min }$. Figure 2 compares the implemented $\Delta z_{s}$ against $\Delta z_{s, \text { min }}$, given by Eq. 5 with $b=5$, for three values of $\Delta x$. It shows that only the $\Delta x=15 \mathrm{~m}$ resolution satisfies Eq. 5 with $b=5$. An initial sensitivity study, not reported here, has revealed that the results from simulations using the $\Delta x=15-\mathrm{m}$ and $30-\mathrm{m}$ resolutions are qualitatively the same. Therefore the lower and computationally less expensive horizontal resolution of $30 \mathrm{~m}$, resulting in 402 and 82 staggered grid points in the $x$ - and $y$-directions, respectively, was used to generate the results reported in Sect. 3. The even number of horizontal grid points enabled the model to be symmetric about its mass points. 


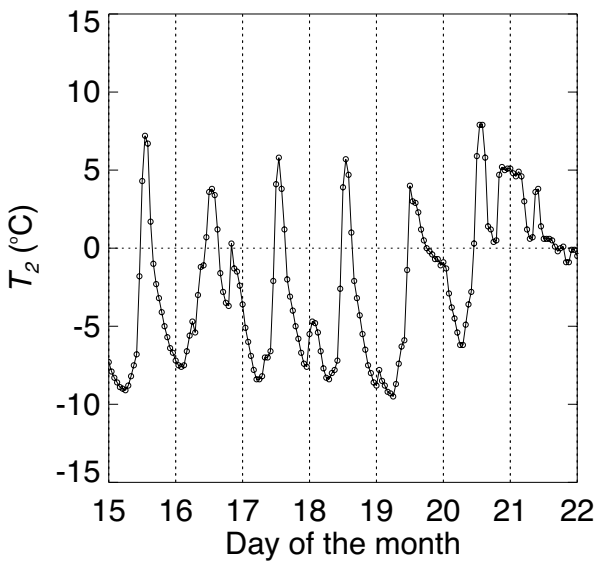

Fig. 3 Time series of 2-m air temperature above the valley floor of the Chamonix valley for a week during January 2003. The time series has a temporal resolution of $1 \mathrm{hr}$. Vertical dotted lines mark the start of each day (0000 UTC). The measurements were taken by Météo France during the POllution in Alpine Valleys (POVA) field campaign (see Brulfert et al. 2005).

\subsection{Initial and boundary conditions}

The simulation was provided with an initial weakly-stable linear lapse rate in virtual potential temperature, $\partial \theta_{v} / \partial z=1.5 \mathrm{~K} \mathrm{~km}^{-1}$, an environmental lapse rate in temperature, $\Gamma$, slightly less than the adiabatic rate. Therefore the simulation represents cases where there is no pre-existing residual layer, or inversions, in the valley atmosphere at the start of the night, indicative of well-mixed post-convective conditions. The model is run for an 8-hr period starting at 1430 UTC on 21 December (that is about $1 \mathrm{hr}$ before sunset at the latitude of the Chamonix valley). The atmosphere at the bottom of the idealized valley was assigned an initial $\theta_{v}=288 \mathrm{~K}$, a temperature of approximately $279.3 \mathrm{~K}$ (about $6{ }^{\circ} \mathrm{C}$ ). This temperature value was chosen to approximately match measurements made in the Chamonix valley, at a similar time of day and year (see Fig. 3). The time rate of change of the 2-m air temperature at the centre of the valley was compared to that of the data in Fig. 3. To make the comparison fair, only the clear-sky observations were considered (i.e., excluding day 19 to 21), and those that did not experience any large shift in the synoptic wind direction (i.e., excluding day 16 and 18). The observed cooling rate averaged over the common time period and over days 15 and 17 is $-1.2 \mathrm{~K} \mathrm{hr}^{-1}$. The corresponding model value is $-0.9 \mathrm{~K} \mathrm{hr}^{-1}$, which given the idealized model set-up, is remarkably similar. The atmosphere was initialized with a spatially constant relative humidity of $40 \%$, which resulted in a moisture flux at the ground/air interface, but avoided any condensation of water in the atmosphere.

The temperature of the ground/air interface, or skin temperature, is initialized by extrapolation of the air temperature of the first three layers above the ground. A random negative thermal perturbation to the extrapolated skin temperatures, with a maximum value of $0.05 \mathrm{~K}$, was applied at the initial time across the valley slopes. This reduced the spin-up time of the simulation, important given the short simulated time period, enforced by the computationally expensive integrations. The thermal perturbation also made the flow three-dimensional (3D), which would not otherwise have been the case, due to the $y$-independent valley geometry, the initial zero wind field (see below), no Coriolis effects, and the otherwise $y$-independent thermal forcing at the surface. Since turbulence is 3D it is important that the flow is 3D too.

For a deep valley under stable conditions, the valley atmosphere is often decoupled from the air above the valley (see, for instance, Whiteman 2000), and so no synoptic forcing was prescribed. It is not known what the velocity field should be within the idealized valley, since this is the problem under investigation, and imposing a zero velocity field within the 
valley space and some non-zero velocity field above would likely lead to unrealistic results. Model grid nesting is beyond the scope of this work, and so the wind field was set to zero everywhere at the initial time.

The model deep soil temperature, at a depth of $8 \mathrm{~m}$ (denoted by $T_{\text {deep }}$ ), is at the maximum depth of penetration of the annual solar temperature wave. A depth of $8 \mathrm{~m}$ for $T_{\text {deep }}$ is reasonable for a soil with low to medium thermal diffusivity, typical of sand-free clay soils, as used for this work (see below). $T_{\text {deep }}$ at the bottom of the valley was set to the annual mean surface air temperature of $281.4 \mathrm{~K}$ (that is $8.25^{\circ} \mathrm{C}$ ), a typical value for a mid-latitude Alpine site at this altitude. This proxy value is regarded as an accurate approximation, based on the premise that long-term thermal equilibrium exists between the soil and atmosphere (e.g. Oke 1987; Chen and Dudhia 2001). Green and Harding (1980) have shown, from numerous measurements across western and central Europe, that the gradient of soil temperature with altitude, during winter, is on average approximately $2 \mathrm{~K} \mathrm{~km}^{-1}$, and that the differences between the gradients in these regions are not large. Gradients were calculated by considering station pairs, with one element of the pair on low ground and the other on high ground. It was found in an earlier study (Green and Harding 1979) that the differences in soil temperature, observed between such a pair of stations, are dominated by the effects of altitude, provided that the height difference between them exceeds $200 \mathrm{~m}$, and that they are in a broadly similar climatic regime. This was found to be the case despite large differences in terrain type, ranging from humus to broken rock. All of the station pairs used by Green and Harding (1980) satisfied the above two criteria. The study also suggests that the gradients of soil temperature with altitude are similar for different soil depths (considering soil depths of 0.1, 0.5 and $1 \mathrm{~m}$ ), and that this pattern generally extends throughout the year. This is important given that the soil temperature measurements were made at different depths, between 0.2 and $1 \mathrm{~m}$, although most were made at $0.5 \mathrm{~m}$. This also suggests that the vertical soil temperature profile does not change greatly with altitude, and so not with changes in average annual temperature, which varies with altitude. The $2 \mathrm{~K} \mathrm{~km}^{-1}$ gradient was used to vary $T_{\text {deep }}$ with altitude across the idealized terrain.

Given a known skin and deep soil temperature the shape of the temperature variation between these boundary values was sought. A linear variation would be easy to implement, however, a better approximation is to increase the temperature exponentially with depth, which is what can generally be observed during the winter months. This general exponential shape can be attributed to the near exponential decay with depth of the surface heat waves, which drive the system about a mean value, in a near periodic fashion. Hillel (1982) showed that the variation of soil temperature with depth and time, due to an infinitely periodic surface heat wave, assuming a constant thermal diffusivity, is of the form

$$
T(z, t)=\left\langle T_{0}\right\rangle+A_{0} e^{z / d} \sin \left(2 \pi f t+\frac{z}{d}+\phi\right)
$$

where $z \leq 0,\left\langle T_{0}\right\rangle$ is the mean soil surface temperature, $A_{0}$ represents the amplitude of the surface wave, $f$ is the wave frequency and $\phi$ is the signal phase shift. The parameter $d$ is the damping depth, given by $d=\sqrt{D_{h} /(f \pi)}$, where $D_{h}$ is the (constant) thermal diffusivity.

Since the sinusoidal variation was applied for an infinite time, there is no transient part to Eq. 6; the soil at any depth is synchronized to the surface signal, or in other words, the soil is in a quasi-steady state. This is not an accurate representation when the surface forcing is applied for some finite time, where the different depths exhibit a transient and more complex behaviour. However, for many systems the quasi-steady state approximation is reasonable, as exemplified by the analysis of Droulia et al. (2009). This model was extended by Droulia 
et al. (2009) to account for both the daily and annual heat waves, which involves the superposition of two waves. The final solution is essentially a sum of terms of the form of Eq. 6. A simplified version of Eq. 6 is introduced that still captures the essential exponential increase of soil temperature with depth,

$$
T(z)=A+B e^{z / d},
$$

where $z \leq 0$ and $d$ was chosen as one third the depth of $T_{\text {deep }}$, with the boundary conditions $T(z=0)=T_{0}$, and $T(z=-3 d)=T_{\text {deep }}$, to give

$$
T(z)=T_{0}+\frac{T_{\text {deep }}-T_{0}}{1-e^{-3}}\left(1-e^{z / d}\right),
$$

where $z \leq 0$. The depth $d$ was chosen to avoid any step in the model output variables during the start of the simulation, which is indicative of a system close to a state of partial equilibrium. This is important since the short simulated period makes long-term model adjustments impractical. The solution was then a compromise between the need to simplify the problem and the need to model the soil temperature profile in a reasonable way.

The idealized terrain was initially set-up to represent an Alpine landscape consisting mainly of grasses, and so the vegetation and landuse type was set to 'grassland', giving, for winter, a surface albedo of 0.23 , a surface emissivity of 0.92 , an aerodynamic roughness length of $0.10 \mathrm{~m}$, and a surface moisture availability of 0.3 (volume fraction).

The soil type was set to 'silty clay loam', a relatively moist soil (Oke 1987), with dry, wilting point, field capacity and maximum soil moistures of $0.120,0.120,0.387$ and 0.464 (volume fractions), respectively. It typically takes a couple of days for a soil to reach its field capacity, after drainage of water via the soil macropores following a rain event, the exact time period depending on the soil properties, the initial water content of the soil, and the initial water depth in the soil. Before the field capacity is reached the gravitational and capillary forces, which dominate the movement (redistribution) of soil water during infiltration and drainage, are both directed downwards. When the field capacity is reached, matric potential or water content gradients are in opposite directions in the upper and lower portions of the soil profile, preventing any significant net downward water flux (Nachabe 1998). Once the macropores are emptied, further drainage, by evaporation from the soil surface or through extraction by plants, removes water at a much slower rate from the soil micropores (Rowell 1994). The method was to provide the soil with a constant soil moisture value $10 \%$ below the chosen soil field capacity, thereby placing the soil safely within the latter soil water redistribution regime. The simulation therefore considered a soil a few days after rainfall, which is reasonable given the winter period modelled, when frequent precipitation is typical in the Alps. The exact soil moisture profile is a complex problem and it is acknowledged that soil moistures are likely to decrease by small amounts with depth, however, over the subdiurnal time period of interest any exchange of moisture between soil layers is negligible.

The model was run with periodic lateral boundary conditions. This was made possible by the relatively large extent of the flat plateaus in the $x$-direction and the $y$-independent valley geometry. If the valley geometry were not $y$-independent then any valley flow is expected to evolve in the down-valley direction precluding the use of a periodic boundary condition in this direction. The implemented valley geometry effectively eliminated any significant valley flow from the results. Without considering a far larger domain, pressure-induced flow cannot be represented. A 4-km deep implicit Rayleigh damping layer (Klemp et al. 2008) was implemented at the top of the model domain to prevent any significant wave reflections affecting the solution. The damping coefficient was set to $0.2 \mathrm{~s}^{-1}$. 

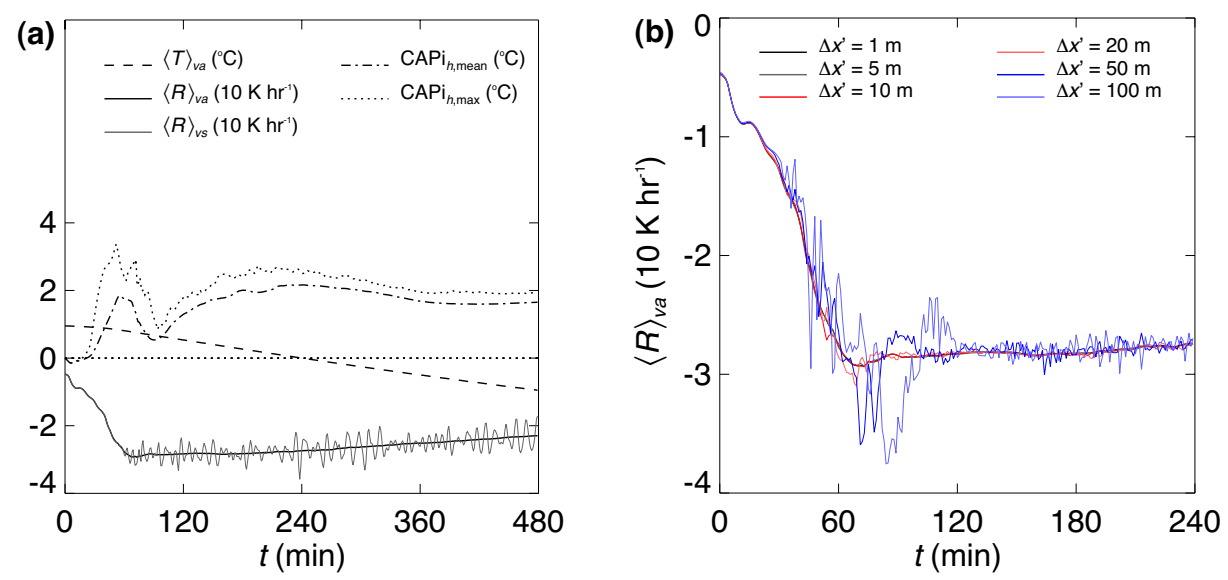

Fig. 4 Time series of (a) average valley-atmosphere temperature, denoted by $\langle T\rangle_{v a},\langle R\rangle_{v a} \equiv\left\langle\partial \theta_{v} / \partial t\right\rangle_{v a}$, where $\theta_{v}$ is virtual potential temperature, $\partial \theta_{v} / \partial t$ averaged over an $((x, z)$ slice taken half-way along $y$, denoted by $\langle R\rangle_{v s}, \mathrm{CAPi}_{h, \text { mean }}$ and $\mathrm{CAPi}_{h, \text { max }}$ (see text for details), and (b) $\langle R\rangle_{v a}$ using a number of different Cartesian grid resolutions.

\section{Results and discussion}

\subsection{Valley-averaged variation of cold-air-pooling processes}

Time series, starting about $1 \mathrm{hr}$ before sunset, of average valley-atmosphere temperature and cooling rate, denoted by $\langle T\rangle_{v a}$, and $\langle R\rangle_{v a} \equiv\left\langle\partial \theta_{v} / \partial t\right\rangle_{v a}$, respectively, where $\partial \theta_{v} / \partial t \approx$ $\partial T / \partial t$ (not shown), are displayed in Fig. 4a. The time series were created by first averaging the model output across the $y$-dimension. The output fields on the model curvilinear grid were then interpolated onto a linear orthogonal framework (i.e., Cartesian system), which filled the two-dimensional valley space, with $\Delta x^{\prime}=\Delta z^{\prime}=5 \mathrm{~m} . \partial \theta_{v} / \partial t$ was calculated from the model output $\theta_{v}$ field using centered finite differencing before re-gridding, to avoid numerical artifacts. The results are not sensitive to the choice of differencing scheme. A local bilinear interpolation was used, that relies on grid indexes, avoiding errors close to sloping ground associated with triangulation techniques using real heights. Such errors are caused by the maximum grid distortion in these regions, together with the rapidly changing fields when moving in a direction normal to the ground. Having said this, $\langle R\rangle_{v a}$ has a low sensitivity to such errors (not shown). The sensitivity of the series to the new grid resolution, was tested using $\partial \theta_{v} / \partial t$ (see Fig. 4b), which demonstrates a convergence of the results as the resolution is increased from 100 to $1 \mathrm{~m}$. Figure $4 \mathrm{~b}$ shows that there is no noticeable difference between the 1 and $5 \mathrm{~m}$ results, and therefore all of the \langle\rangle$_{v a}$ and \langle\rangle$_{v s}$ time series were created using the latter resolution, where the subscript $v s$ stands for a valley section, in the $(x, z)$ plane. Errors occurring in $\langle R\rangle_{v a}$, as the resolution is decreased, are primarily caused by the misrepresentation of the valley atmosphere away from the valley slopes.

Figure 4a shows a general steady cooling of the valley atmosphere through time. $\langle T\rangle_{v a}$ is approximately $1{ }^{\circ} \mathrm{C}$ for the first $30 \mathrm{~min}$ or so of simulation, before decreasing at a near constant rate of roughly $0.25 \mathrm{~K} \mathrm{hr}^{-1}$, to reach a final value close to $-1{ }^{\circ} \mathrm{C}$, revealing a total $2{ }^{\circ} \mathrm{C}$ decrease of $\langle T\rangle_{v a}$ during the simulated 8-hr period. The initial near-constant temperature is due to a balance between cooling from longwave radiation and combined heating from shortwave radiation and dynamical processes, when all terms are small. The change in $\langle T\rangle_{v a}$ 
over this period is $-0.043 \mathrm{~K}$ and in $\left\langle\theta_{v}\right\rangle_{v a}$ is $-0.046 \mathrm{~K}$. In general, the subsequent decrease of $\langle T\rangle_{v a}$ is caused by the reduction and loss of shortwave radiation effects, an increase in the cooling from longwave radiation, and the initiation of cooling from dynamical processes, as discussed in this section. The associated instantaneous cooling rate, $\langle R\rangle_{v a}$, initially increases in magnitude relatively quickly, due to the changes in the forcing mechanisms, with $\langle R\rangle_{v a}$ decreasing from -0.048 to $-0.29 \mathrm{~K} \mathrm{hr}^{-1}$ at $t=73 \mathrm{~min} .\left|\langle R\rangle_{v a}\right|$ then gradually decreases with $\langle R\rangle_{v a}$ increasing to $-0.23 \mathrm{~K} \mathrm{hr}^{-1}$ at the end of the simulation. During this latter period there is a general reduction in the cooling from longwave radiation and dynamics effects. Possible oscillatory features can be seen in $\langle R\rangle_{v s} \equiv\left\langle\partial \theta_{v} / \partial t\right\rangle_{v s}$, taken halfway along the $y$-dimension, after $t=60 \mathrm{~min}$ (see Fig. 4a). These features are discussed in the Appendix.

\subsubsection{Cold-air-pool intensity}

Due to the relatively large valley depth and possibly the chosen initial stratification, the simulated temperatures over the plateaus are always less than the temperatures over the valley floor, despite the enhanced cooling there, and in this respect a CAP is not simulated. This highlights the ambiguity that remains in the definition of a CAP, which typically refers to the relatively low air temperatures in a volume of air confined towards the bottom of a depression, compared to a reference air temperature above it. This work has found evidence of slightly higher temperatures immediately above the shallow (less than 100-m deep) layer of air at the bottom of the valley (not shown). However, the sign of this temperature difference is quickly reversed by moving the reference further away from this layer. The approach taken here was to remove the hydrostatic variation in temperature from all points in the model domain, allowing for a comparison of model domain cooling rates to those over flat terrain at the same elevation. This revealed a region of enhanced cooling that expanded upwards from the bottom of the valley (see Sect. 3.2.1), denoted by $\mathrm{CAP}_{h}$, where the subscript $h$ refers to the hydrostatic adjustment. The CAP intensity (CAPi), has therefore been denoted by $\mathrm{CAPi}_{h} . \mathrm{CAPi}_{h}$ was calculated in two ways: as the difference between the model adjustedplateau and valley floor average near-surface air temperatures, denoted by $\mathrm{CAPi}_{h \text {,mean }}$, and also using the adjusted-maximum and minimum values from the two respective regions, denoted by $\mathrm{CAPi}_{h, \text { max }}$. All temperatures were taken from the model first mass points at 0.76 and $0.81 \mathrm{~m}$ above the plateaus and valley floor, respectively (i.e., approximately at screen-level height). For the first $15 \mathrm{~min}$ both $\mathrm{CAPi}_{h}$ curves show negative values, that is the plateaus initially cooled faster than the valley floor (see Fig. 4a). The maximum magnitude of the temperature difference is small, with $\mathrm{CAPi}_{h \text {,mean }}=-0.15^{\circ} \mathrm{C}$. After $t=15 \mathrm{~min} \mathrm{CAPi}_{h \text {,mean }}$ and $\mathrm{CAPi}_{h, \text { max }}$ become positive and remain so for the remainder of the simulation, highlighting the enhanced cooling at the bottom of the valley compared to air adjacent to flat terrain in the stable decoupled conditions. Immediately after $t=15 \mathrm{~min}$ there is a peak in both curves centered close to $t=60 \mathrm{~min}$, before $\mathrm{CAPi}_{h \text {,mean }}$ and CAPi ${ }_{h, \text { max }}$ increase again at a progressively decreasing rate until about $t=240 \mathrm{~min}$. Both $\mathrm{CAPi}_{h}$ curves then gradually decrease until approximately $t=360 \mathrm{~min}$ before levelling off for the remainder of the simulation, suggesting that some form of equilibrium or partial equilibrium condition was reached. The maximum $\mathrm{CAPi}_{h, \text { max }}$ is $3.4{ }^{\circ} \mathrm{C}$ at $t=52 \mathrm{~min}$, in contrast to the maximum $\mathrm{CAPi}_{h \text {, mean }}$ of $2.2^{\circ} \mathrm{C}$ at $t=228 \mathrm{~min}$.

\subsubsection{Cold-air-pool forcing mechanisms}

Figure 5a reveals that the \langle\rangle$_{v a}$ accumulated temperature change due to net radiation only, $\left\langle\Delta \theta_{v_{r}}\right\rangle_{v a}$, is fairly uniform, and reaches a total value close to $-1 \mathrm{~K}$, where the subscript 

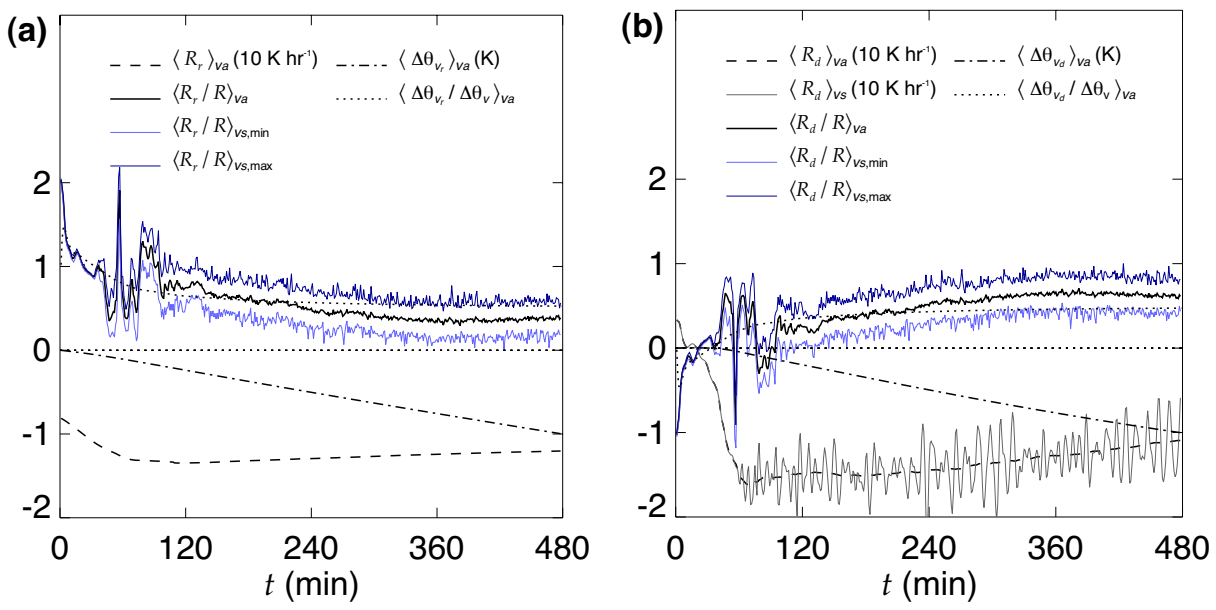

Fig. 5 Time series of (a) the average valley-atmosphere radiative part of $\partial \theta_{v} / \partial t \equiv R$, denoted by $\left\langle R_{r}\right\rangle_{v a}$, $\left\langle R_{r} / R\right\rangle_{v a},\left\langle R_{r} / R\right\rangle_{v s, \text { min }}$ and $\left\langle R_{r} / R\right\rangle_{v s, \text { max }}$ obtained by using the operator \langle\rangle$_{v s}$ for every $y$ position and searching across $y$ at each time for the minimum and maximum $\left\langle R_{r} / R\right\rangle_{v s},\left\langle\Delta \theta_{v_{r}}\right\rangle_{v a}$, where $\Delta \theta_{v_{r}}$ is the accumulated change of $\theta_{v}$ due to net radiation, $\left\langle\Delta \theta_{v_{r}} / \Delta \theta_{v}\right\rangle_{v a}$, where $\Delta \theta_{v}$ is the total accumulated change of $\theta_{v}$, and (b) the same as (a) but considering dynamics quantities, as well as $\left\langle R_{d}\right\rangle_{v s}$ taken half-way along $y$.

$r$ is short for radiation. $\left\langle\Delta \theta_{v_{r}}\right\rangle_{v a}$ at $t=30 \mathrm{~min}$ is small with a value of $-0.047 \mathrm{~K}$. Also shown is the \langle\rangle$_{v a}$ cooling rate due to net radiation, denoted by $\left\langle R_{r}\right\rangle_{v a}$, where $\left\langle R_{r}\right\rangle_{v a} \approx$ $\left\langle(\partial T / \partial t)_{r}\right\rangle_{v a}$ (not shown). Initially $\left|\left\langle R_{r}\right\rangle_{v a}\right|$ increases relatively rapidly with $\left\langle R_{r}\right\rangle_{v a}$ decreasing from -0.081 to $-0.13 \mathrm{~K} \mathrm{hr}^{-1}$ at $t=65 \mathrm{~min}$, before decreasing again only slightly to a rate of $-0.135 \mathrm{~K} \mathrm{hr}^{-1}$ at $t=113 \mathrm{~min}$. After this time $\left|\left\langle R_{r}\right\rangle_{v a}\right|$ decreases gradually with $\left\langle R_{r}\right\rangle_{v a}$ increasing to $-0.12 \mathrm{~K} \mathrm{hr}^{-1}$ at the end of the simulation. Figure 5a displays the \langle\rangle$_{v a}$ contribution of $\Delta \theta_{v_{r}}$ and $R_{r}$ to the total quantities, denoted by $\left\langle\Delta \theta_{v_{r}} / \Delta \theta_{v}\right\rangle_{v a}$ and $\left\langle R_{r} / R\right\rangle_{v a}$, respectively. The averaging must be done after the normalization to correctly represent the normalized model output fields. $\left\langle\Delta \theta_{v_{r}} / \Delta \theta_{v}\right\rangle_{v a}$ increases for the first 5 min from approximately 102 to $148 \%$, before decreasing relatively rapidly to $77 \%$, at $t=65 \mathrm{~min}$, after which it generally decreases at a progressively slower rate to reach a final value of $53 \%$. The initial increase of $\left\langle\Delta \theta_{v_{r}} / \Delta \theta_{v}\right\rangle_{v a}$ balances the heating caused by dynamics effects (see Fig. 5b). $\left\langle\Delta \theta_{v_{r}} / \Delta \theta_{v}\right\rangle_{v a}$ completely dominates the cooling for the initial $30 \mathrm{~min}$, with $\left\langle\Delta \theta_{v_{r}} / \Delta \theta_{v}\right\rangle_{v a}=103 \%$ at $t=30 \mathrm{~min}$, and the subsequent rapid decrease of $\left\langle\Delta \theta_{v_{r}} / \Delta \theta_{v}\right\rangle_{v a}$ accounts for the growing influence of the dynamics in the total temperature changes. Figure $5 \mathrm{~b}$ illustrates the difference between the total and radiation fields (i.e., the combined dynamics effects of advection and subgrid-scale turbulent mixing) for which the subscript $d$ is used. $\left\langle\Delta \theta_{v_{d}}\right\rangle_{v a}$ is near zero for the first $30 \mathrm{~min}$ of the simulation, in fact amounting to a very small positive temperature change of $0.00062 \mathrm{~K}$. Over the same period shortwave radiation caused a temperature change, $\left\langle\Delta \theta_{v_{S W}}\right\rangle_{v a}$, of $0.00525 \mathrm{~K}$ (see Fig. 6), as expected to give $\left\langle\Delta \theta_{v}\right\rangle_{v a} \cdot\left|\left\langle\Delta \theta_{v_{d}}\right\rangle_{v a}\right|$ then increases steadily to a final value close to $-1 \mathrm{~K}$, as expected.

$\left\langle R_{d}\right\rangle_{v a}$ initially decreases from 0.033 to $-0.16 \mathrm{~K} \mathrm{hr}^{-1}$ at $t=73 \mathrm{~min}$, changing from a small heating rate to a relatively large cooling rate. $\left|\left\langle R_{d}\right\rangle_{v a}\right|$ then generally decreases with $\left\langle R_{d}\right\rangle_{v a}$ increasing to a final value of $-0.11 \mathrm{~K} \mathrm{hr}^{-1}$. The oscillatory features in $\left\langle R_{d}\right\rangle_{v s}$ (see Fig. 5b) could not be found in the data for $\left\langle R_{r}\right\rangle_{v s}$, which reveals that the oscillations in $\langle R\rangle_{v s}$ (see Fig. 4a) are caused by the dynamics alone. $\left\langle\Delta \theta_{v_{d}} / \Delta \theta_{v}\right\rangle_{v a}$ first decreases from -2 to $-48 \%$ at $t=5 \mathrm{~min}$, before increasing rapidly until approximately $t=75 \mathrm{~min}$, reaching 


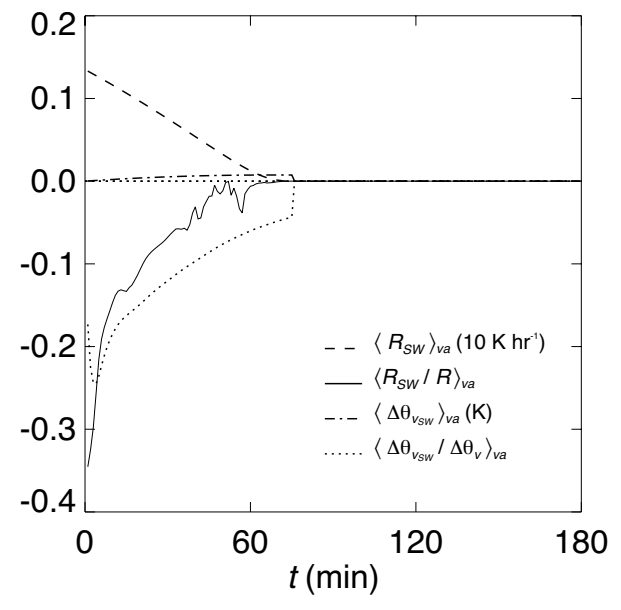

Fig. 6 Time series of $\left\langle R_{S W}\right\rangle_{v a}, \quad\left\langle R_{S W} / R\right\rangle_{v a}$, $\left\langle\Delta \theta_{v_{S W}}\right\rangle_{v a}$ and $\left\langle\Delta \theta_{v_{S W}} / \Delta \theta_{v}\right\rangle_{v a}$, where $R_{S W}$ and $\Delta \theta_{v_{S W}}$ are the instantaneous and accumulated changes of $\theta_{v}$ due to shortwave radiation.

$27 \%$, and then increasing only gradually to reach a final value of $47 \%$. The initial heating effect by the dynamics and the subsequent time it takes for the cooling by the dynamics to take effect, together with broadly similar rates of cooling from longwave radiation and dynamics, once the flow is established, results in $\left\langle\Delta \theta_{v_{d}} / \Delta \theta_{v}\right\rangle_{v a}<\left\langle\Delta \theta_{v_{r}} / \Delta \theta_{v}\right\rangle_{v a}$. The two forcing mechanisms are tightly coupled, and ultimately it is longwave radiation that causes the downslope flows (discussed in Sect. 3.2.1). It would be interesting to investigate further the generality of this result, for instance by varying the initial conditions.

$\left\langle R_{r} / R\right\rangle_{v a}$ and $\left\langle R_{d} / R\right\rangle_{v a}$ are more variable than $\left\langle\Delta \theta_{v_{r}} / \Delta \theta_{v}\right\rangle_{v a}$ and $\left\langle\Delta \theta_{v_{d}} / \Delta \theta_{v}\right\rangle_{v a}$, and are formed of three fairly distinct regimes (see Fig. 5). As above, there is first a 30-min period when longwave radiation almost completely dominates the cooling, with $\left\langle R_{r} / R\right\rangle_{v a}$ decreasing from 203 to $90 \%$. After approximately $t=30 \mathrm{~min}$ there is a period of instability, lasting some $90 \mathrm{~min}$, where $\left\langle R_{r} / R\right\rangle_{v a}$ is of course exactly out of phase with $\left\langle R_{d} / R\right\rangle_{v a}$. Finally, there is a gradual reduction of $\left\langle R_{r} / R\right\rangle_{v a}$ from $75 \%$ to a final contribution of $37 \%$. Figure 5 also gives the maximum and minimum values of $\left\langle R_{r} / R\right\rangle_{v s}$ and $\left\langle R_{d} / R\right\rangle_{v s}$, by applying \langle\rangle$_{v s}$ to every $y$-position and searching across $y$ at each time. Both plots demonstrate that there is little variation from $\left\langle R_{r} / R\right\rangle_{v a}$ and $\left\langle R_{d} / R\right\rangle_{v a}$ for about the first $30 \mathrm{~min}$ of the simulation, suggesting that the thermodynamics are constrained to develop in an essentially two-dimensional way during this period. The variation around $\left\langle R_{r} / R\right\rangle_{v a}$ and $\left\langle R_{d} / R\right\rangle_{v a}$ is generally larger during the period of instability, depending on the specific time considered. After $t=120 \mathrm{~min}$, the variation around $\left\langle R_{r} / R\right\rangle_{v a}$ and $\left\langle R_{d} / R\right\rangle_{v a}$ is near constant over time, with the volume averages close to the centre of the variation defined be the maximum and minimum values (defining a maximum variation of about $40 \%$ ). The simulation average for $\left\langle\Delta \theta_{v_{r}} / \Delta \theta_{v}\right\rangle_{v a}$ and $\left\langle R_{r} / R\right\rangle_{v a}$ is 64 and $58 \%$, respectively. The corresponding values for the period of gradual decline are 56 and $46 \%$. The maximum (minimum) values for $\left\langle\Delta \theta_{v_{r}} / \Delta \theta_{v}\right\rangle_{v a}$ and $\left\langle R_{r} / R\right\rangle_{v a}$ are 147 (53) and $203(29) \%$, respectively. The times of these percentages are respectively 5 (480) and 1 (382) min. Figure 6 shows that shortwave radiation has only a small modulating influence in the first hour or so of the simulation, decreasing the cooling due to net radiation, and increasing the rate of initial increase of $\left|\left\langle R_{r}\right\rangle_{v a}\right|$. Hoch et al. (2011) found values of about $30 \%$ for $\left\langle\Delta \theta_{v_{L W}} / \Delta \theta_{v}\right\rangle_{v a}$ and $\left\langle R_{L W} / R\right\rangle_{v a}$, early in the night, from 1700 to $2200 \mathrm{LST}$, for the Arizona meteor crater, which is clearly different from the respective values of 56 and $46 \%$ obtained for the period of gradual decline, from 1630 to 2230 UTC. One possible 
explanation is an over-estimation of $\left\langle R_{L W} / R\right\rangle_{v a}$ by the one-dimensional radiative transfer scheme used for the simulation.

The RRTM longwave radiation scheme, used here, does not consider photon transport between atmospheric columns, and so nor reflections or emissions from surrounding terrain. The work by Hoch et al. (2011) using the MYSTIC code, a 3D radiative transfer model, demonstrated that one-dimensional schemes will tend to over-estimate \langle\rangle$_{v a}$ longwave radiative cooling rates, denoted by $\left\langle R_{L W}\right\rangle_{v a}$ hereafter (see Fig. 6 and 7 in Hoch et al. 2011). The 1900 LST MYSTIC simulation suggests there will be an error close to $0.05 \mathrm{~K} \mathrm{hr}^{-1}$ in the $t=270$ min (1900 UTC) $\left|\left\langle R_{L W}\right\rangle_{v a}\right|$ value reported here, where $\left\langle R_{L W}\right\rangle_{v a}$ is always negative. The 1900 LST MYSTIC simulation had a similar bulk atmospheric temperature profile to the 1900 UTC WRF model results from this work (not shown). Hoch et al. (2011) assumed a temperature deficit of $4 \mathrm{~K}$, which is larger than the corresponding value of about $2.5 \mathrm{~K}$, for this work, obtained half-way up the western valley slope and considering the temperature change across the downslope flow, which is less than 50-m deep (not shown). However, the MYSTIC model results suggests that this is unlikely to have any significant effect on the error. Hoch et al. (2011) made simulations for 1500, 1900 and 0600 LST, which revealed that the error is not constant in time. Although the WRF simulation made here, began at a similar time to the first MYSTIC simulation, the initial conditions were different, which makes even any linear approximation of the changing error impossible. This would nevertheless make an interesting topic of future research. Also, in the present work, the shortwave radiation decreased at approximately the same rate everywhere, since slope effects on shortwave radiation were not included. Including shadowing effects is likely to cause a different initiation of the flow (e.g. Lehner et al. 2011), a subject of future work.

\subsection{Local-scale features}

\subsubsection{Cold-air-pool evolution}

Details of the valley-atmosphere cooling are difficult to appreciate in the $T$ or $\theta_{v}$ fields, due to the hydrostatic change of these quantities with $z$. However, both fields show the general cooling and stabilization of the valley atmosphere as the night progresses. The $T$ or $\theta_{v}$ fields also indicate that in general the valley atmosphere cooling is horizontally homogeneous. This effect is also indicated by the $\Delta \theta_{v}$ field and gives a clearer picture of the cooling variation across the domain. Figure 7 displays filled contour plots of $\left\langle\Delta \theta_{v}\right\rangle_{y}$ overlaid with streamlines. The streamlines were created by tracing the paths of massless particles through $\left\langle\mathbf{u}_{x z}\right\rangle_{y}$, by time integration, where $\mathbf{u}$ is the model wind field and $\mathbf{u}_{x z} \equiv(u, w)$. Each 'particle' was tracked from its seed point until the path left the input space or a maximum number of iterations was reached. After adding arrows at the seed points to reveal flow direction, the approach has the advantage of indicating the relative strength, direction and vorticity of the flow, across the input space. The filled contours were created using a 5-m grid resolution, justified above for the field averages, and the streamline seed points are positioned on the Cartesian grid with the same origin, with $\Delta x^{\prime}=\Delta z^{\prime}=100 \mathrm{~m}$, placing a limit on the range of turbulent scales that can be revealed in these particular plots. Nevertheless, it was found that the displayed streamlines are a good representation of the streamlines generated from a finer seed-point mesh. The streamline algorithm uses $\left\langle\mathbf{u}_{x z}\right\rangle_{y}$ projected onto the 5-m grid to track the 'particle' trajectories from the seed points. $u$ is the dominant component of $\mathbf{u}$, which together with the implemented idealized terrain and initial conditions, suggests that the major features of the flow exist in the $(x, z)$ plane, and so the streamlines are a good 
representation of the dominant flow features. To give an idea of the absolute magnitudes of the flow in the following analysis, it should be noted that the established downslope flow has a typical speed of approximately $2 \mathrm{~m} \mathrm{~s}^{-1}$.

Close inspection of Fig. 7a reveals that the dark region immediately above the top half of the western valley slope, no more than 50-m deep, is the region of maximum flow, which hides a corresponding region of relatively large accumulated temperature decrease, with $\left\langle\Delta \theta_{v}\right\rangle_{y} \approx-1 \mathrm{~K}$, compared to the surrounding atmosphere that has $-0.1<\left\langle\Delta \theta_{v}\right\rangle_{y}<0 \mathrm{~K} . \Delta \theta_{v}$ is negative everywhere at all times. Figures $7 \mathrm{a}$ and $7 \mathrm{~b}$ reveal a propagating intensification of the downslope flow, with a counter-clockwise vortex at the head of this flow, considering a northerly oriented rotation axis. The downslope flow was found to exist, albeit to a lesser degree, from within $5 \mathrm{~min}$ of $t=0$ (not shown). There is evidence of relatively large cooling at the bottom of the valley at $t=40 \mathrm{~min}$, with $-0.2<\left\langle\Delta \theta_{v}\right\rangle_{y}<-0.1 \mathrm{~K}$ within about $100 \mathrm{~m}$ above ground level, and with $\left\langle\Delta \theta_{v}\right\rangle_{y} \leq-2 \mathrm{~K}$ within a few meters of the valley floor. This cooling is due to a combination of radiation and dynamics effects. The intensification of the downslope flow is shown to generally disturb the quiescent valley atmosphere, creating further vortices away from the terrain, a general upward motion close to the valley axis, which is to be expected due to mass conservation, as well as a movement of air towards the slope behind the vortex at the head of the maximum flow region. Despite the variability in the system, this latter counter-clockwise half-valley-scale circulation becomes a quasipermanent feature of the valley flow system. Figure $7 \mathrm{c}$ shows the beginning of the reflection of the maximum flow region back towards the bottom of the slope, after colliding with the fluid from the eastern slope. A small-scale eddy about 100-m across, close to the centre of the valley, indicates the presence of turbulence in a shallow region less than 100-m deep.

Soon after $t=60$ min the signature of internal gravity waves (IGWs) becomes clear, in and above the valley atmosphere (see Fig. 7d), which supports the evidence of IGWs reported in the Appendix. The general direction of the wave vector $\left\langle\mathbf{k}_{x z}\right\rangle_{y}$, where $\mathbf{k}_{x z} \equiv\left(k_{x}, k_{z}\right)$, at this time, is clear, revealed by the upward and downward streamline regions, with $\left\langle\mathbf{k}_{x z}\right\rangle_{y}$ directed westwards to allow for an upward energy propagation. The streamlines indicate that $\left\langle\mathbf{k}_{x z}\right\rangle_{y}$ makes an angle of about $30^{\circ}$ with the vertical, which agrees with $0.88<\left\langle\omega^{\prime} / N\right\rangle_{x z}<0.92$ (see the Appendix), and that $2 \pi /\left\langle k_{z}\right\rangle_{y}=\left\langle\lambda_{z}\right\rangle_{y} \approx 1 \mathrm{~km}$, which are very similar to the results of Chemel et al. (2009) and Largeron et al. (2013), and supports their finding that $\lambda_{z}$ is set by the depth of the topography. An interesting feature of the flow are the vortices between the regions of upward and downward motions. A full description of the IGW field is beyond the scope of this work.

Figure $7 \mathrm{~d}$ also shows the further retreat of the maximum flow region back towards the bottom of the slope, which leaves behind it a region of relatively large $\left|\left\langle\Delta \theta_{v}\right\rangle_{y}\right|$ air, indicating the importance of the downslope flow for the valley bottom cooling in the early night. The downslope flow intensification mixes the region of large $\left|\left\langle\Delta \theta_{v}\right\rangle_{y}\right|$ at the bottom of the valley higher into the atmosphere. By $t=120 \mathrm{~min}$ (see Fig. 7e), the maximum flow region has retreated further, with a clear deflection of the downslope flow, close to the bottom of the slope, as it comes into contact with air of a similar or greater density. Figures $7 \mathrm{f}$ and $7 \mathrm{~g}$ show the further growth of the $\mathrm{CAP}_{h}$ and subsequent retreat of the downslope flow maximum region back up the western slope. The streamlines in these latter plots were made white for clarity, however, the apparent loss of the IGW signature is deceiving. From $t=120 \mathrm{~min}$ onwards, streamlines run westward beginning close to the centre of the valley in a near-horizontal region approximately $100-\mathrm{m}$ deep, positioned about $100 \mathrm{~m}$ above the plateau height (see Fig. $7 \mathrm{f}$ and $7 \mathrm{~g}$ ), and develop together with a valley atmosphere capping inversion (not shown). This flow feature is linked to the quasi-permanent counter-clockwise flow system noted above. 

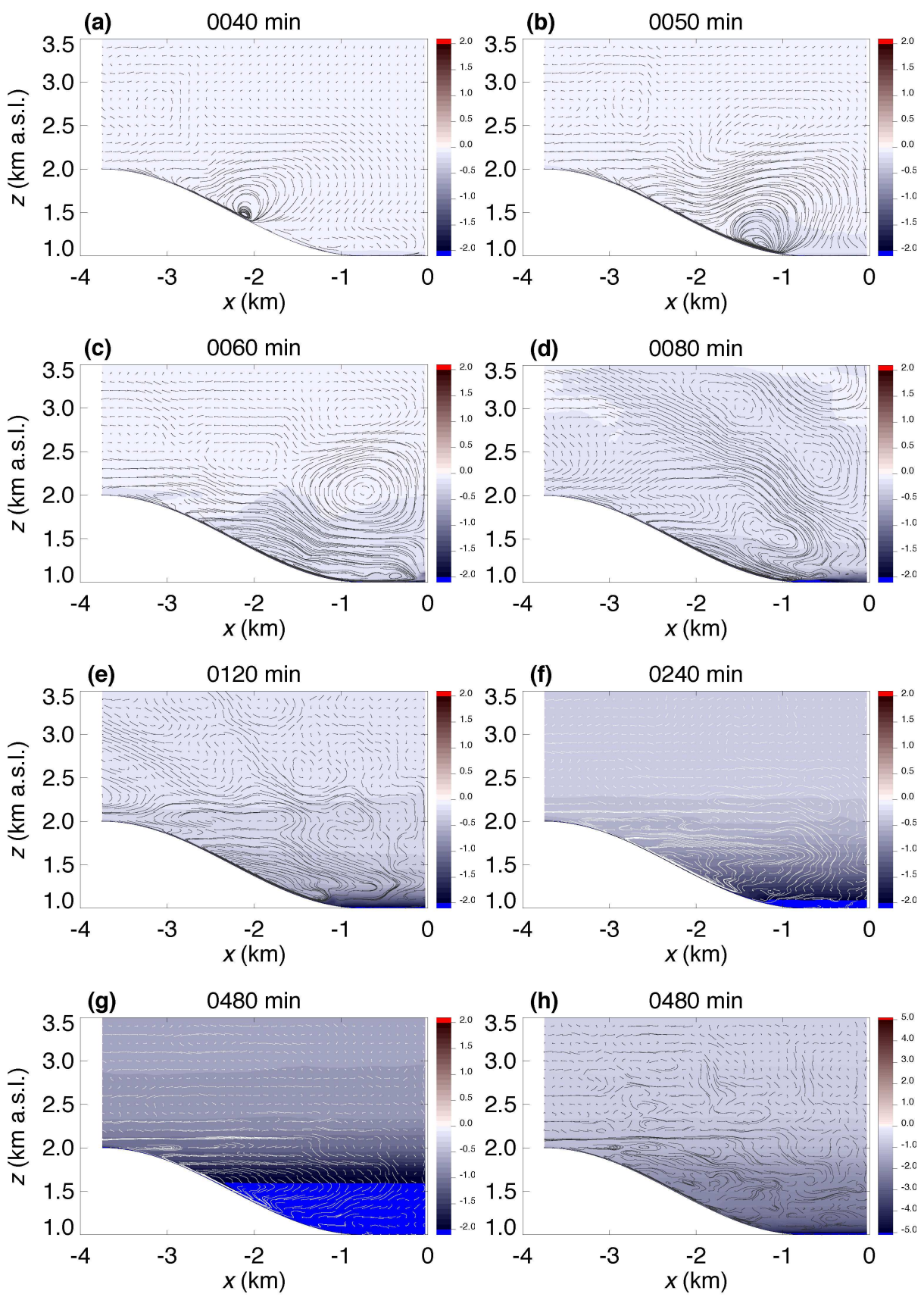

Fig. 7 Contour plots of (a) to (g) $\left\langle\Delta \theta_{v}\right\rangle_{y}$ (in K), with solid black or white streamlines over-plotted at $t=40$, 50, 60, 80, 120, 240 and $480 \mathrm{~min}$, and (h) $\Delta \theta_{v}$ (in K) taken half-way along the $y$-dimension at $t=480 \mathrm{~min}$. 
The return flow above the downslope flow over the bottom half of the slope is clear in Fig. 7f, revealed by the S-shaped streamlines adjacent to the ground. There is evidence of flow separation above the developing $\mathrm{CAP}_{h}$ during the early night (see Fig. $7 \mathrm{~d}$ to $7 \mathrm{f}$ ), though the feature is difficult to see later in the night, when there is clear evidence of flow penetration into the developing $\mathrm{CAP}_{h}$ (see Fig. 7g). The colour scale in Fig. 7 a through $7 \mathrm{~g}$ was chosen to make clear the development of the $\mathrm{CAP}_{h}$, however, the detail of the cooling at the very bottom of the valley is lost after $t=120 \mathrm{~min}$. Figure $7 \mathrm{~h}$ indicates the relatively large cooling effect within the first $100 \mathrm{~m}$ of the valley bottom, compared to the atmosphere above, the effect intensifying as the night progresses. Streamlines for an $(x, z)$ slice of the domain, taken half-way along the $y$-dimension, reveals the localized variability in the dynamics along $y$. The dominant flow features are still apparent. However, the turbulent nature of the flow is more clear, and it would be interesting to investigate further the exact mixing characteristics of the valley atmosphere, as well as the ability of the valley-flow system to mix scalars into the free atmosphere.

\subsubsection{Cold-air-pool forcing mechanisms}

Figure 8 shows contour plots of $\left\langle R_{r} / R\right\rangle_{y}$, with streamlines over-plotted, as above. $R$ is found to have both signs, whereas, $R_{r}$ is always negative, with the exception of a few rare cases of radiative heating at the very bottom of the valley atmosphere (not shown). As pointed out above, the air temperatures are always less than at $t=0$, despite the occasional heating rate. This means that, in general, $\left\langle R_{r} / R\right\rangle_{y}>1$ corresponds to a cooling atmosphere due to radiative processes, despite heating from the dynamics, and $\left\langle R_{r} / R\right\rangle_{y}<0$ corresponds to a heating atmosphere due to the dynamics overcoming radiative cooling. A clockwise circulating vortex, with rotation axis into the page, can be seen in Fig. 8a, centered at approximately $(x=-3.1 \mathrm{~km}, z=2.75 \mathrm{~km})$. The region of bright red colour on the eastern edge of this vortex corresponds to $\left\langle R_{r} / R\right\rangle_{y}>1$, and the streamlines suggest this is caused by the downward advection of air from about $z=3 \mathrm{~km}$. $\Gamma$ is less than the dry adiabatic rate, denoted by $\Gamma_{d}$, everywhere at $t=0$, and in this region $\Gamma$ decreases slightly with time, however, downward advected parcels of air will experience compressional warming at $\Gamma_{d}$, since there is no liquid water in the atmosphere. This will result in warmer parcels displacing cooler ones and $\left\langle R_{d}\right\rangle_{y}>0$. Evidently the heating from the dynamics is not large enough to overcome the radiative cooling in this case. The opposite effect can be seen on the western side of the vortex where, $0<\left\langle R_{r} / R\right\rangle_{y}<1$, due to $\left\langle R_{d}\right\rangle_{y}<0$, due to the expansion and cooling of parcels as they rise higher through the atmosphere, adding to the radiative cooling. The patterns in $R$ generally correspond to those in $R_{d}$, which is expected given the uniformity of $R_{r}$ (not shown). $R_{d}<0$ corresponds to enhanced total cooling, whereas $R_{d}>0$ corresponds to reduced total cooling or a warming (that is $R>0$ ). As well as the absence of liquid water, these compressional effects rely on $\Gamma<\Gamma_{d}$ (i.e., a stable atmosphere), and overturning and mixing is implied whenever $\Gamma>\Gamma_{d}$, which occurs close to the ground at times towards the valley bottom (not shown). $\Gamma$ is near constant in space and time above $z=2.5 \mathrm{~km}$, where the main cause of cooling variability is reversible compression effects, potentially affected by the horizontal advection of air. Below $z=2.5 \mathrm{~km}$, where the dynamics is controlled by the downslope flows, the sources of cooling variability are more complex, as further explained below.

The 'blue' region positioned mainly behind the largest vortex, at the front of the downslope flow maximum region, indicating $\langle R\rangle_{y}>0$ and $\left\langle R_{d}\right\rangle_{y}>0$, is likely caused, at least in part, by compressional effects, as above. The 'blue' region corresponds to the area where the streamlines indicate the maximum downward transport of air. Close inspection of the 

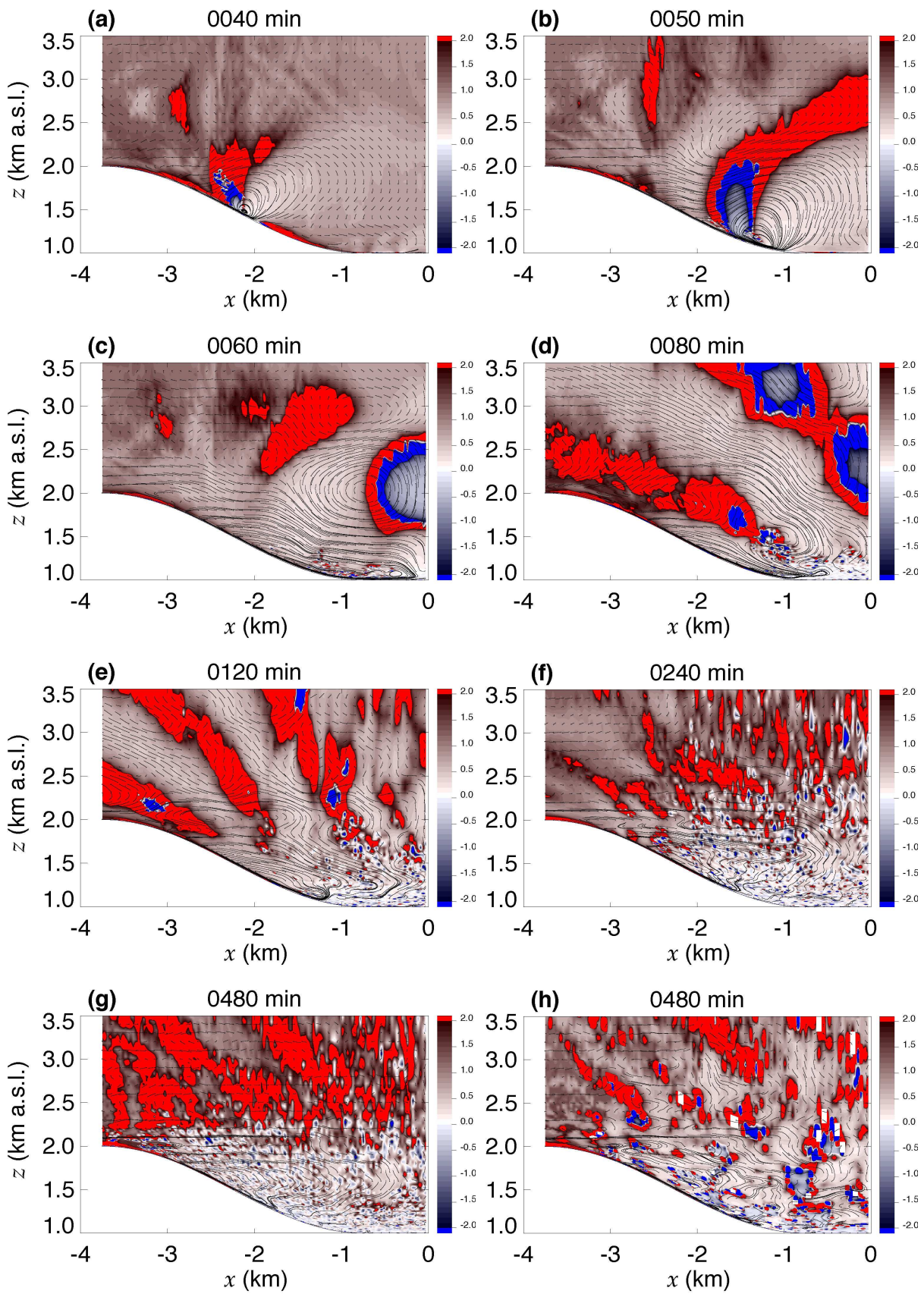

Fig. 8 Contour plots of (a) to (g) $\left\langle R_{r} / R\right\rangle_{y}$, with black streamlines over-plotted at $t=40,50,60,80,120,240$ and $480 \mathrm{~min}$, and (h) $R_{r} / R$ taken half-way along the $y$-dimension at $t=480 \mathrm{~min}$. 
field immediately above the valley floor reveals that the enhanced cooling here, noted above, is due to a combination of radiation and dynamics effects. Figures $8 \mathrm{~b}$ and $8 \mathrm{c}$ correspond roughly to the times of minimum and maximum $\left\langle R_{r} / R\right\rangle_{v a}$, during the period of instability, shown in Fig. 5a, at $t=47$ and $57 \mathrm{~min}$, with values of 35 and $191 \%$, respectively. The cause of these extreme values is now clear. Considering the valley atmosphere only, Fig. $8 \mathrm{~b}$ shows a greater upward transport of air together with a larger 'blue' region, compared to Fig. 8c, where the streamlines have been generally tilted towards the horizontal and the 'blue' region, carried with the flow, has been partly forced upwards and out of the valley atmosphere by the colliding opposite flows. The relatively intense upward motion occurs when the downslope flow intensification reaches the bottom of the slope. The situation is perhaps similar to the minimum $9 \%$ radiative cooling rate contribution found by Hoch et al. (2011) during a midnight air-intrusion into the Arizona meteor crater. Interestingly, the time of minimum $\left\langle R_{r} / R\right\rangle_{v a}$, during the period of instability, occurs only $5 \mathrm{~min}$ before the time of maximum $\mathrm{CAPi}_{h, \max }$.

The large changes in $\left\langle R_{r} / R\right\rangle_{y}$ occurring over small distances adjacent to the valley floor, at $t=60 \mathrm{~min}$, in general, are well correlated with nearby unstable air, which complements the evidence of turbulence in this region provided by the small-scale eddy, noted above. An animation of an $(x, z)$ slice of $R_{r} / R$, taken half-way along $y$, reveals that the smallest of these turbulent features generally originate from the front of the downslope flow maximum region and are transported down the slopes towards the valley centre. This effect is not clear in $\left\langle R_{r} / R\right\rangle_{y}$, after approximately $t=80 \mathrm{~min}$ (when the variability across $y$ increases), due to the averaging operation, which makes the analysis of small-scale features difficult. Figures $8 \mathrm{~d}$ and 8e show clearly that $\left\langle\mathbf{k}_{x z}\right\rangle_{y}$ tilts towards the ground as the waves move closer to the plateau, which agrees well with the analysis of $\left\langle\omega^{\prime} / N\right\rangle_{x z}$, also revealed in the patterns of $\omega^{\prime}$ (see the Appendix). The thermodynamics effects of the IGWs are clear, with regions of $\left\langle R_{d}\right\rangle_{y}>0$ occurring in the downward streamline regions of the waves, caused by counterrotating vortices between the upward streamline regions, where $\left\langle R_{d}\right\rangle_{y}<0$. Close inspection of the $(x, z)$ slices, as above, for all time, has revealed a general westward movement of regions with reduced cooling, or $\langle R\rangle_{y}>0$, that are inter-spaced by regions of enhanced cooling, over the top half of the slope. Analysis of the streamlines indicates that in many cases these features are caused by westward propagating vortices, together with their associated compressional effects, as explained above. The instances where no vortex can be found reveal the occurrence of near-laminar advective effects. The westward transport of heating effects and vortices is caused by the quasi-permanent anti-clockwise circulation, and shallow region of near-horizontal streamlines close to the plateau height. Many of the heating features are absorbed into the downslope flow region. Higher above the plateaus there is also an apparent westward movement of compressional heating and cooling regions, but in fact this effect is due to the propagating IGWs that modulate the flow. Figures $8 \mathrm{f}$ and $8 \mathrm{~h}$ provide some evidence of these effects. Figure $8 \mathrm{~h}$ is for an $(x, z)$ slice, providing a representation of the turbulent flow field, as well as suggesting the continued presence of IGWs above the valley atmosphere at the end of the simulated period. Figures $8 \mathrm{~d}$ through $8 \mathrm{~g}$ make clear the dominance of cooling by the dynamics within the developing $\mathrm{CAP}_{h}$ (see Fig. 7). Both radiation and dynamics effects appear to be important for the upward expansion of $\mathrm{CAP}_{h}$. The general existence of relatively small-scale effects above the $\mathrm{CAP}_{h}$ suggests that the cold-airpooling processes cause an interaction between the valley air and the free atmosphere above, although the degree of this effect remains unclear.

The minimum $R_{r}$ within the valley atmosphere is $-3.19 \mathrm{~K} \mathrm{hr}^{-1}$, occurring immediately adjacent to the ground. Generally, the greatest cooling in $R_{r}$ is adjacent to the ground, with 
$R_{r}$ decreasing steadily with distance from the surface (not shown). In comparison, Hoch et al. (2011) found a maximum cooling rate of $-1.25 \mathrm{~K} \mathrm{hr}^{-1}$ close to the ground surface.

\section{Summary}

The purpose of this work was to unravel the physical processes controlling cold-air pools in complex terrain. For this purpose, the WRF numerical model was used to examine the variation of key cold-air-pooling forcing mechanisms in an idealized alpine-valley domain with a width of order $10 \mathrm{~km}$ under decoupled stable conditions.

The total average valley-atmosphere cooling results from a complex balance/interplay between radiation and dynamics effects. There are three fairly distinct regimes in the evolution of cold-air-pooling processes. Starting about $1 \mathrm{hr}$ before sunset, there is an initial 30-min period when the downslope flows are initiated and longwave radiation almost completely dominates the cooling. A period of instability follows, when there is a competition between radiation and dynamics effects, lasting some $90 \mathrm{~min}$. Finally, there is a gradual reduction of the contribution of radiative cooling to total average valley-atmosphere cooling, $\left\langle R_{r} / R\right\rangle_{v a}$, from $75 \%$ to a final contribution of $37 \%$. The maximum cold-air-pool intensity corresponds to the time when cooling by radiation effects is at a minimum, within the period of instability. The initial heating effect by the dynamics and the subsequent time it takes for the dynamics effects to cool the valley atmosphere, together with broadly similar rates of cooling from radiation and dynamics, once the flow is established, results in $\left\langle\Delta \theta_{v_{d}} / \Delta \theta_{v}\right\rangle_{v a}<\left\langle\Delta \theta_{v_{r}} / \Delta \theta_{v}\right\rangle_{v a}$.

Further work is needed to investigate further the generality of this result, for example, by varying the initial conditions. The simulation average is approximately $64 \%$ for $\left\langle\Delta \theta_{v_{r}} / \Delta \theta_{v}\right\rangle_{v a}$, and $56 \%$ for the period of gradual decline. For the latter time period, Hoch et al. (2011) found a value of about $30 \%$ for $\left\langle\Delta \theta_{v_{L W}} / \Delta \theta_{v}\right\rangle_{v a}$. One possible explanation of the difference is the overestimation of radiative heat loss by the one-dimensional radiative transfer scheme used for this work, even though the effects of different terrain geometries and initial conditions considered by the two studies can not be ruled out.

Some of the intricacies of the valley mixing have been revealed. There are places where the dynamics dominate the cooling and radiation effects are minor.

Internal gravity waves have been identified in and above the valley atmosphere. An analysis of $\omega^{\prime}$ complements the work of Chemel et al. (2009) and Largeron et al. (2013). It has been found that $0.88<\left\langle\omega^{\prime} / N\right\rangle_{x z} \approx\left\langle\omega^{\prime}\right\rangle_{x z} /\langle N\rangle_{x z}<0.92$ for $-2.25 \leq x \leq 2.25 \mathrm{~km}, 2.5 \leq z \leq$ $3.5 \mathrm{~km}$ a.s.1., and $0.80<\left\langle\omega^{\prime} / N\right\rangle_{x z} \approx\left\langle\omega^{\prime}\right\rangle_{x z} /\langle N\rangle_{x z}<0.835$ for $-3.75 \leq x \leq 3.75 \mathrm{~km}$, with the same $z$ range. The difference is caused by lower values of $\omega^{\prime}$ above the top of the valley slopes, associated with a tilting of the wave vector towards the ground as the waves approach the plateaus, also apparent in the streamlines. The ratios decrease with $t$, as $N$ increases, in agreement with the findings of Largeron et al. (2013).

\section{Appendix}

It is clear that $\langle R\rangle_{v s}$ satisfies the Dirichlet conditions, and so a Fourier series will converge to the signal. A fast Fourier transform (FFT) of form $g_{\omega}=1 / N_{p} \sum_{n=0}^{N_{p}-1} f_{n} e^{2 \pi \omega n / N_{p}}$, with $\omega=0, \ldots, N_{p}-1$, was applied to $\langle R\rangle_{v s}$, taken half-way along $y$, after $t=60 \mathrm{~min}$, where $\omega$ is the discrete set of angular frequencies, and $N_{p}$ the number of points in the discrete time series $f_{n}$. Figure 9 a reveals that the harmonic with $\omega$ close to $0.01 \mathrm{rad} \mathrm{s}^{-1}$, a period close to 

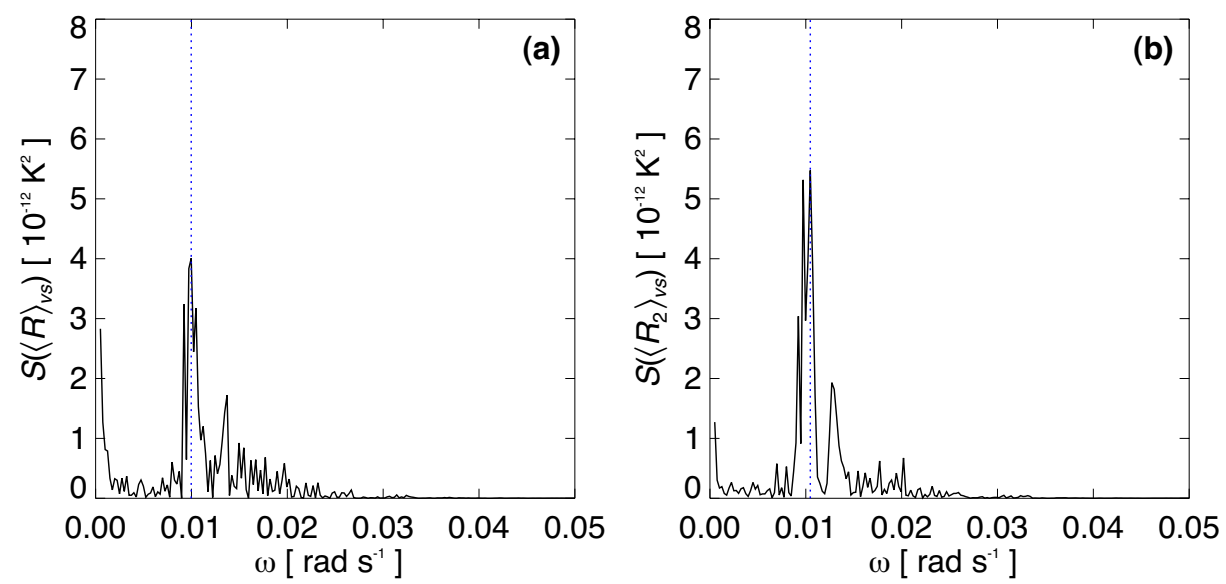

Fig. 9 Spectrum of the time series, after $t=60 \mathrm{~min}$, of (a) $\langle R\rangle_{v s}$ and (b) $\left\langle R_{2}\right\rangle_{v s}$, taken half-way along $y$, where the vertical dotted lines mark the frequencies with the largest amplitude in the spectrum (see text for details).

10.48 min (see Fig. 4a), dominated the signal, with $2\left|g_{\omega}\right|^{2}=S\left(\langle R\rangle_{v s}\right)=4.0110^{-12} \mathrm{~K}^{2}$. The zero frequency, the signal mean, was removed, as was the fundamental frequency, which is statistically not well defined and otherwise dominated the signal. The FFT normalizes $g_{\omega}$ by $N_{p}$, in order that Parseval's theorem applies, that is, in discrete form, $1 / N_{p} \sum_{n=0}^{N_{p}-1}\left|f_{n}\right|^{2}=$ $\sum_{\omega=0}^{N_{p}-1}\left|g_{\omega}\right|^{2}$, where the total energy of the signal is the same in both the real and phase-space domains. For a real signal $\sum_{\omega=0}^{N_{p}-1}\left|g_{\omega}\right|^{2}=\sum_{\omega=0}^{N_{p} / 2-1} 2\left|g_{\omega}\right|^{2}$. An in-depth spectral analysis is beyond the scope of this work, however, it is reasonable to argue that the dominant peak, found above, is the signature of internal gravity waves (IGWs). It was confirmed that the oscillations in $\langle R\rangle_{v s}$ are caused by the dynamics and not radiative processes (see Sect. 3.1.2).

All points in the $\partial \theta_{v} / \partial t$ field within $100 \mathrm{~m}$ of the sloping valley sidewalls were then removed and \langle\rangle$_{v s}$ was applied to the resulting field, in the same way as for $\left\langle\partial \theta_{v} / \partial t\right\rangle_{v s}$, which provided a time series, denoted by $\left\langle R_{2}\right\rangle_{v s}$, free from any signature of an oscillating downslope flow. Figure 9b displays the above FFT applied to $\left\langle R_{2}\right\rangle_{v s}$, which, when compared to Fig. 9a, shows that the oscillations in $\langle R\rangle_{v s}$ are likely the result of IGWs propagating through the stable valley atmosphere. Largeron et al. (2013) demonstrated that it is the unstable and/or oscillatory downslope flow that initiates the IGWs. Further investigation, using the dominant frequency identified above, denoted by $\omega_{\left\langle R_{2}\right\rangle_{v s}}^{\prime}$, where $\omega^{\prime}$ denotes the frequency with the largest amplitude in the spectrum of a time series, considering all times after $t=60 \mathrm{~min}$, is difficult since $\left\langle R_{2}\right\rangle_{v s}$ does not provide an accurate representation of the wave field (not shown). The problem is due to the averaging operation rather than the chosen proxy variable, which is representative of the wave field for positions above $200 \mathrm{~m}$ from the terrain surface (see Fig. 10). The symmetry of the terrain and initial conditions makes the model output qualitatively symmetric about the valley axis, and therefore only the western side of the valley atmosphere is presented to make clear any features of interest.

An initial analysis of $\omega^{\prime}$ across an $(x, z)$ slice taken half-way along $y$ (see Fig. 10a) indicates that the wave field within the valley atmosphere is non-uniform. The reasons behind this heterogeneity have not been fully quantified, but seem likely to include wavewave interactions (Largeron et al. 2013), which strictly precludes the use of IGW linear theory in this region, as well as the use of a single representative $\omega^{\prime}$ for the valley atmo- 


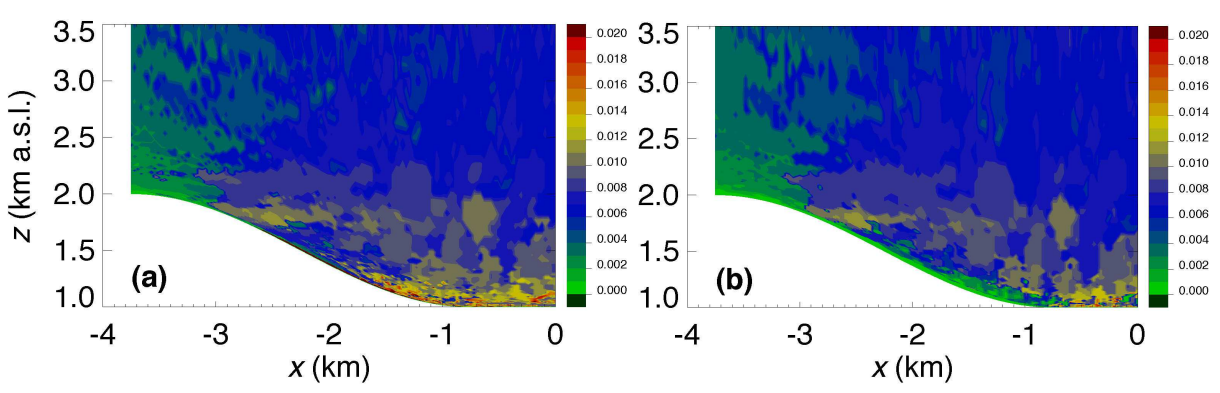

Fig. 10 Contour plots of $\omega^{\prime}$ (in $\mathrm{rad} \mathrm{s}^{-1}$ ), the frequency with the largest amplitude in the spectrum of the time series, after $t=60 \mathrm{~min}$, of (a) $\partial \theta_{v} / \partial t$ and (b) $w$, across an $(x, z)$ slice taken half-way along $y$.

sphere. $\omega^{\prime}$, as defined above, is not required to be a clearly dominant frequency, and an initial analysis indicates that the dominance of $\omega^{\prime}$ is less clear wherever there is a relatively large and rapid spatial change in $\omega^{\prime}$ (not shown). It is interesting that the waves in the valley atmosphere, above $200 \mathrm{~m}$ from the terrain surface, are restricted to approximately $0.005 \leq \omega^{\prime} \leq 0.014 \mathrm{rad} \mathrm{s}^{-1}$. The wave field above $z=2.5 \mathrm{~km}$ a.s.l. (i.e., $500 \mathrm{~m}$ above the plateaus), and for $-2.25 \leq x \leq 2.25 \mathrm{~km}$ (between the slope inflection points), is quasimonochromatic (see Fig. 10a), which permits the use of a single representative $\omega^{\prime}$. Using a similar model set-up to that used here, Chemel et al. (2009) found $\omega^{\prime} / N_{0} \approx 0.8-0.9$ at two locations a few hundred metres above the valley atmosphere, where $N_{0}$ is the Brunt-Väisälä frequency at $t=0$. The sensitivity study by Largeron et al. (2013) extended the work by Chemel et al. (2009) and found $0.8<\left\langle\omega^{\prime}\right\rangle_{y} / N_{0}<0.9$ for a similar location above the valley atmosphere, where \langle\rangle$_{y}$ indicates an average across $y$. These results were found to correspond to IGWs radiated by any turbulent field with no dominant frequency component. For the model set-up used here, $N$ is near-constant in space above $2.5 \mathrm{~km}$ a.s.1., for the full simulated period (not shown), resulting in $0.88<\left\langle\omega^{\prime} / N\right\rangle_{x z} \approx\left\langle\omega^{\prime}\right\rangle_{x z} /\langle N\rangle_{x z}<0.92$, with the ratio decreasing with $t$ as $N$ increases slightly. The averages were made across $-2.25 \leq x \leq 2.25 \mathrm{~km}$, $2.5 \leq z \leq 3.5 \mathrm{~km}$ a.s.l., where the upper $z$ limit was chosen to lie well below the Rayleigh damping layer at $8 \mathrm{~km}$ a.s.l. Largeron et al. (2013) also found the ratio to generally decrease with increasing $N$. Extending the $x$ range to include the regions of lower $\omega^{\prime}$ above the top of each slope, with $-3.75 \leq x \leq 3.75 \mathrm{~km}$, gives $0.80<\left\langle\omega^{\prime} / N\right\rangle_{x z} \approx\left\langle\omega^{\prime}\right\rangle_{x z} /\langle N\rangle_{x z}<0.835$. The two ranges of $\left\langle\omega^{\prime} / N\right\rangle_{x z}$ correspond reasonably well with those reported by Chemel et al. (2009) and Largeron et al. (2013).

\section{References}

Anquetin S, Guilbaud C, Chollet JP (1998) The formation and destruction of inversion layers within a deep valley. J Appl Meteorol 37:1547-1560

Anquetin S, Guilbaud C, Chollet JP (1999) Thermal valley inversion impact on the dispersion of a passive pollutant in a complex mountainous area. Atmos Environ 33:3953-3959

Brulfert C, Chemel C, Chaxel E, Chollet JP (2005) Modelling photochemistry in alpine valleys. Atmos Chem Phys 5:2341-2355

Catalano F, Cenedese A (2010) High-resolution numerical modeling of thermally driven slope winds in a valley with strong capping. J Appl Meteorol Climatol 49:1859-1880

Chazette P, Couvert P, Randriamiarisoa H, Sanak J, Bonsang B, Moral P, Berthier S, Salanave S, Toussaint F (2005) Three-dimensional survey of pollution during winter in French Alps valleys. Atmos Environ 39:1035-1047 
Chemel C, Staquet C, Largeron Y (2009) Generation of internal gravity waves by a katabatic wind in an idealized alpine valley. Meteorol Atmos Phys 103:187-194

Chen F, Dudhia J (2001) Coupling an advanced land-surface/hydrology model with the Penn State/NCAR MM5 modeling system. Part I: model implementation and sensitivity. Mon Weather Rev 129:569-585

Chen F, Zhang Y (2009) On the coupling strength between the land surface and the atmosphere: From viewpoint of surface exchange coefficients. Geophys Res Lett 36:L10,404, DOI 10.1029/2009GL037980

Cheng Y, Brutsaert W (2005) Flux-profile relationships for wind speed and temperature in the stable atmospheric boundary layer. Boundary-Layer Meteorol 114:519-538

Daly C, Conklin DR, Unsworth MH (2010) Local atmospheric decoupling in complex topography alters climate change impacts. Int J Climatol 30:1857-1864

Deardorff JW (1980) Stratocumulus-capped mixed layers derived from a three-dimensional model. BoundaryLayer Meteorol 18:495-527

Doran JC, Horst TW (1983) Observations and models of simple nocturnal slope flows. J Atmos Sci 40:708717

Droulia F, Lykoudis S, Tsiros I, Alvertos N, Akylas E, Garofalakis I (2009) Ground temperature estimations using simplified analytical and semi-analytical approaches. Solar Energy 83:211-219

Dudhia J (1989) Numerical study of convection observed during the winter monsoon experiment using a mesoscale two-dimensional model. J Atmos Sci 46:3077-3107

Dudhia J (1995) Reply. Mon Weather Rev 123:2573-2575

Fairall CW, Bradley EF, Rogers DP, Edson JB, Young GS (1996) Bulk parameterization of air-sea fluxes for Tropical Ocean Global Atmosphere Coupled-Ocean Atmosphere Response Experiment. J Geophys Res 101:3747-3764

Green FHW, Harding RJ (1979) The effects of altitude on soil temperature. Meteorol Mag 108:81-91

Green FHW, Harding RJ (1980) Altitudinal gradients of soil temperatures in Europe. Trans Inst Br Geogr 5:243-254

Grisogono B, Kraljevic L, Jericevic A (2007) The low-level katabatic jet height versus Monin-Obukhov height. Q J Roy Meteorol Soc 133:2133-2136

Gustavsson T, Karlsson M, Bogren J, Lindqvist S (1998) Development of temperature patterns during clear nights. J Appl Meteorol 37:559-571

Helmis CG, Papadopoulos KH (1996) Some aspects of the variation with time of katabatic flow over simple slope. Q J Roy Meteorol Soc 122:595-610

Hillel D (1982) Introduction to Soil Physics. Academic Press, New York, NY, USA, 364 pp

Hoch SW, Whiteman DC, Mayer B (2011) A systematic study of longwave radiative heating and cooling within valleys and basins using a three-dimensional radiative transfer model. J Appl Meteorol Climatol 50:2473-2489

Iacono MJ, Delamere JS, Mlawer EJ, Shephard MW, Clough SA, Collins WD (2008) Radiative forcing by long-lived greenhouse gases: calculations with the AER radiative transfer models. J Geophys Res 113:D13,103, DOI 10.1029/2008JD009944

Jiménez PA, Dudhia J, Gonzalez-Rouco JF, Navarro J, Montávez JP, García-Bustamante E (2012) A revised scheme for the WRF surface layer formulation. Mon Weather Rev 140:898-918

Klemp JB, Dudhia J, Hassiotis AD (2008) An upper gravity-wave absorbing layer for NWP applications. Mon Weather Rev 136:3987-4004

Lacis AA, Hansen JE (1974) A parameterization for the absorption of solar radiation in the Earth's atmosphere. J Atmos Sci 31:118-133

Largeron Y, Staquet C, Chemel C (2013) Characterization of oscillatory motions in the stable atmosphere of a deep valley. Boundary-Layer Meteorol 148:439-454

Lehner M, Whiteman D, Hoch SW (2011) Diurnal cycle of thermally driven cross-basin winds in Arizona's meteor crater. J Appl Meteorol Climatol 50:729-744

Lundquist KA (2010) Immersed boundary methods for high-resolution simulation of atmospheric boundarylayer flow over complex terrain. PhD thesis, University of California, Berkeley, CA, USA, 159 pp

Mahrer Y (1984) An improved numerical approximation of the horizontal gradients in a terrain-following coordinate system. Mon Weather Rev 112:918-922

Mansell ER, Ziegler CL, Bruning EC (2010) Simulated electrification of a small thunderstorm with twomoment bulk microphysics. J Atmos Sci 67:171-194

Mayer B (2009) Radiative transfer in the cloudy atmosphere. Eur Phys J Conf 1:75-99

Mayer B, Kylling A (2005) The libRadtran software package for radiative transfer calculations: Description and examples of use. Atmos Chem Phys 5:1855-1877

Moeng CH, Dudhia J, Klemp J, Sullivan P (2007) Examining two-way grid nesting for large eddy simulation of the PBL using the WRF model. Mon Weather Rev 135:2295-2311

Nachabe MH (1998) Refining the definition of field capacity in the literature. J Irrigat Drain Eng 124:230-232 
Noppel H, Fiedler F (2002) Mesoscale heat transport over complex terrain by slope winds - a conceptual model and numerical simulations. Boundary-Layer Meteorol 104:73-97

Oke TR (1987) Boundary Layer Climates. Routledge, London, UK, London, UK, 464 pp

Price JD, Vosper S, Brown A, Ross A, Clark P, Davies F, Horlacher V, Claxton B, McGregor JR, Hoare JS, Jemmett-Smith B, Sheridan P (2011) COLPEX: field and numerical studies over a region of small hills. Bull Am Meteorol Soc 92:1636-1650

Rowell DL (1994) Soil Science: Methods \& Applications. Prentice Hall, Upper Saddle River, NJ, USA, $350 \mathrm{pp}$

Scotti A, Meneveau C, Lilly DK (1993) Generalized Smagorinsky model for anisotropic grids. Phys Fluids 5:2306-2308

Sheridan PF, Vosper SB, Brown AR (2013) Characteristics of cold pools observed in narrow valleys and dependence on external conditions. Q J Roy Meteorol Soc DOI 10.1002/qj.2159, in press

Shu CW (2003) High-order finite difference and finite volume WENO schemes and discontinuous Galerkin methods for CFD. Int J Comput Fluid Dyn 17:107-118

Skamarock WC, Klemp JB, Dudhia J, Gill DO, Barker DM, Duda MG, Huang XY, Wang W, Powers JG (2008) A Description of the Advanced Research WRF Version 3. NCAR Technical Note NCAR/TN475+STR, NCAR, Boulder, CO, USA, 125 pp

Skyllingstad ED (2003) Large eddy simulation of katabatic flows. Boundary-Layer Meteorol 106:217-243

Smith CM, Skyllingstad ED (2005) Numerical simulation of katabatic flow with changing slope angle. Mon Weather Rev 133:3065-3080

Smith SA, Brown AR, Vosper SB, Murkin PA, Veal AT (2010) Observations and simulations of cold air pooling in valleys. Boundary-Layer Meteorol 134:85-108

Stephens GL (1978) Radiation profiles in extended water clouds. Part II: Parameterization schemes. J Atmos Sci 35:2123-2132

Szintai B, Kaufmann P, Rotach MW (2010) Simulation of pollutant transport in complex terrain with a numerical weather prediction-particle dispersion model combination. Boundary-Layer Meteorol 137:373-396

Thompson BW (1986) Small-scale katabatics and cold hollows. Weather 41:146-153

Vinokur M (1980) On One-Dimensional Stretching Functions for Finite-Difference Calculations. NASA Contractor Report 3313, NASA, Washington, DC, USA, 56 pp

Vosper SB, Brown AR (2008) Numerical simulations of sheltering in valleys: the formation of nighttime cold-air pools. Boundary-Layer Meteorol 127:429-448

Vosper SB, Hughes JK, Lock AP, Sheridan PF, Ross AN, Jemmett-Smith B, Brown AR (2013) Cold-pool formation in a narrow valley. Q J Roy Meteorol Soc DOI 10.1002/qj.2160, in press

Whiteman CD (2000) Mountain Meteorology: fundamentals and applications. Oxford University Press, New York, NY, USA, $355 \mathrm{pp}$

Zardi D, Whiteman CD (2013) Diurnal mountain wind systems. In: Chow FK, De Wekker SFJ, Snyder BJ (eds) Mountain Weather Research and Forecasting: Recent Progress and Current Challenges, Springer Atmospheric Sciences, Springer, New York, NY, USA, chap 2, pp 35-119 